\title{
ANÁLISIS DEL IMPACTO SOCIOECONÓMICO DE LAS DENOMINADAS 'PIRÁMIDES' EN EL DEPARTAMENTO DEL CAUCA, 2006-2009*
}

\author{
Alejandra Miller Restrepo** \\ Andrés Mauricio Gómez Sánchez***
}

Recibido: marzo 02 de 2010

Aceptado: enero 20 de 2011

\section{RESUMEN}

El objetivo del artículo es evaluar el impacto social y económico de las captadoras ilegales de dinero denominadas "pirámides" en la ciudad de Popayán y el departamento del Cauca, bajo el contexto de la reciente crisis financiera mundial. En este sentido, el documento utiliza inicialmente información secundaria para realizar análisis descriptivos coyunturales, y posteriormente la construcción de dos indicadores basados en mediciones econométricas que incluyen vectores propios para analizar el comportamiento de las captadoras ilegales y el comportamiento de la crisis mundial en la región. Estos son contrastados, y se observa que mientras el país se hundía en la crisis financiera, las pirámides blindaron la economía del Cauca, pero después de sus abruptas caídas, el efecto fue mucho más grave para la economía regional pues el PIB regional disminuyó a niveles más profundos que el PIB Nacional.

\section{PALABRAS CLAVE}

Actividad económica regional, depósitos, micro-intermediarios financieros, microdatos, vectores propios.

\section{CLASIFICACIÓN JEL} R10, G21, G29, C43, C81

\section{CONTENIDO}

Introducción; 1. Contexto económico nacional y regional; 2. El contexto social en el departamento del Cauca; 3. Construcción de indicadores para las pirámides y la crisis financiera: metodología econométrica; 4. Conclusiones; Bibliografía.

\footnotetext{
Este artículo es producto de una investigación de los autores, adscritos al grupo de investigación GICEA (Grupo de investigación en Contabilidad, Economía y Administración) de la Facultad de Ciencias Contables Económicas y Administrativas de la Universidad del Cauca, que se encuentra en el escalafón C de COLCIENCIAS. Surge por la necesidad de conocer el papel que cumplieron las captadoras ilegales de dinero en la actual desestabilización de la economía local-regional, al igual que su trascendencia en aspectos de carácter social. 2009p = Significa año 2009 parcial, porque se presentan algunas evidencias para el primer trimestre del año 2009.

* Economista, Universidad del Valle, Cali, Colombia. Magíster en Ciencias Políticas, Universidad Javeriana, Cali, Colombia. Docente del Departamento de Economía, Universidad del Cauca, Popayán, Colombia. Miembro del Grupo de Investigación GICEA, y coordinadora del Grupo de Estudios de Genero, de la Universidad del Cauca, Colombia. Dirección: Calle 5 No. 4-70, Universidad del Cauca, Popayán, Colombia. Correo electrónico: amiller@unicauca.edu.co.

*** Economista, Universidad del Valle, Cali, Colombia. Especialista, Gerencia de Proyectos, Universidad del Cauca, Popayán, Colombia Magíster en Economía Aplicada, Universidad del Valle, Cali, Colombia. Docente del Departamento de Economía, Universidad del Cauca, Popayán, Colombia. Miembro del Grupo de Investigación GICEA y Polinomía, Universidad del Cauca, Popayán, Colombia. Dirección: Calle 5 No. 4-70, Universidad del Cauca, Popayán, Colombia. Correo electrónico: amgomez@unicauca.edu.co.
} 


\section{ANALYSIS OF THE SO-CALLED PYRAMIDS IN CAUCA DEPARTMENT, 2006-2009}

\section{ABSTRACT}

The objective of this article is to evaluate the social and economic impact of the illegal money collection companies so-called "pyramids" in the city of Popayan and in the lower region of the Cauca Department under the context of a recent world financial crisis. In this sense, the document initially uses secondary information in order to develop a situational descriptive analysis, and hereinafter the construction of two indicators that are based in econometric measurements that include own vectors for analyzing the behavior of the illegal money collection and the behavior of the world economic crisis in the region. These are contrasted and it can be observed that while the country fell into a financial crisis, the pyramids protected the economy of Cauca's department, but after their aggressive falls, the effect was much worse in the regional economy where the regional GDP fell more than the National GDP.

\section{KEY WORDS}

Regional Economic Activity, Deposits, Micro financial intermediaries, Micro data, own vectors.

JEL CLASSIFICATION

R10, G21, G29, C43, C81

\section{CONTENTS}

Introduction; 1. National and Regional Economic Context; 2. The Social context in the Cauca Department. 3. Construction of indicators for the pyramids and the financial crisis: Econometric Methodology 4. Conclusions; Bibliography.

\section{ANALISE DO IMPACTO SOCIOECONÔMICO DAS DENOMINADAS 'PIRÂMIDES' NO DEPARTAMENTO DO CAUCA, 2006-2009}

\section{RESUMO}

O objetivo do artigo é avaliar o impacto social e econômico das captadoras ilegais de dinheiro denominadas 'pirâmides' na cidade do Popayan e o departamento do Cauca baixo o contexto da recente crise financeira mundial. Neste sentido, o documento usa incialmente informação secundaria para fazer analise descritivo conjuntural, e posteriormente a construção de dois indicadores baseados em medições econométricas que incluem vetores próprios para analisar o comportamento das captadoras ilegais e o comportamento da crise mundial na região. Estes são contrastados e pode-se observar que no momento que o país caia numa crise financeira, as pirâmides blindavam a economia da região, más depois das fortes caídas, o efeito foi muito maior para a região que para a economia do país pois o PIB desceu muito mais que o PIB do país.

\section{PALAVRAS-CHAVES}

Atividade econômica regional, Depósitos, Micro intermediários financeiros, Micro dados, vetores próprios.

CLASSIFICAÇÃO JEL R10, G21, G29, C43, C81

\section{CONTEÚDO}

Introdução; 1. Contexto econômico nacional e regional; 2 O contexto social no departamento do Cauca; 3. Construção de indicadores para as pirâmides e a crise financeira; metodologia financeira; 4. Conclusões; Bibliografia. 


\section{INTRODUCCIÓN}

Durante el segundo semestre del 2008 se inició la crisis financiera internacional más profunda desde la Gran Depresión de los años treinta. Simultáneamente, Colombia y, particularmente, el suroccidente del país, fueron el escenario propicio para el nacimiento, la consolidación y el derrumbe de 250 captadoras ilegales de dinero llamadas coloquialmente "pirámides". Este fenómeno tuvo su auge y desplome en el 2008, lo que generó distintos efectos para las economías de las regiones más comprometidas, especialmente Cauca, Nariño y Putumayo. Se calcula que la captadora Dinero Fácil, Rápido y Efectivo (DRFE) captó cerca de dos billones de pesos en 64 oficinas en todo el país, mientras que la captadora David Murcia Guzmán (DMG) obtuvo ingresos de 72 mil millones de pesos en 2007, según la revista Semana (2008). Tal circunstancia creó diferentes consecuencias económicas y sociales en los territorios en donde hubo mayor presencia de este fenómeno. De una parte, en los momentos de más auge de las pirámides, la economía local y regional mantenía liquidez permanente para sostener el consumo, y de otra, el desplome causó efectos graves que profundizaron la desaceleración económica producto de la crisis financiera mundial.

Sobre este escenario, no muy distinto a la realidad de otras ciudades medianas y pequeñas del país, el artículo que se presenta a continuación es producto de una investigación regional sobre el impacto económico y social que generó el derrumbe de las captadoras ilegales de dinero o pirámides en Popayán y el Cauca. La primera y segunda partes contienen una aproximación al contexto económico y social de la región, en la tercera parte del artículo se desarrolla un modelo econométrico que permite hacer el análisis del impacto económico y finalmente se hacen unas aproximaciones a los perfiles de los "inversionistas" de las pirámides y unas conclusiones.

\section{CONTEXTO ECONÓMICO NACIONAL Y REGIONAL}

En la región se hace evidente el impacto de la crisis financiera con la caída del PIB regional'1. Se observa que durante los tres primeros trimestres del 2008, la crisis impactó con mayor severidad al país que a la región, pues durante este período el departamento del Cauca tuvo un crecimiento por encima del PIB Nacional; sin embargo, en la mitad de último trimestre del 2008 se muestra una caída vertiginosa de la economía regional, como se observa en el gráfico 1, mucho más profunda que la disminución en el PIB Nacional, que evidencia el impacto adicional que sobre la región tuvo el desplome de las pirámides.

Dada la ausencia de información económica agregada actualizada para la región, se ha analizado el desempeño económico regional a partir de ciertos Indicadores Líderes de la Actividad Económica, seleccionados por el DANE para analizar el comportamiento de la economía nacional. La selección de indicadores busca presentar un número reducido de variables que permitan sintetizar lo que ocurre en el sector real de la economía caucana, con el fin de anticipar el comportamiento del PIB².

Es importante anotar que una revisión del comportamiento de estos mismos indicadores en el contexto nacional muestra, en su mayoría, un

El PIB Regional trimestral se calculó con metodología ARIMA. Los modelos ARIMA (Procesos Autorregresivos Integrados de Promedios Móviles) son una metodología econométrica que permite pronosticar los valores futuros de una serie o variable económica teniendo en cuenta su comportamiento histórico. La serie bajo análisis debe ser estacionaria, es decir, que su valor promedio debe tener una media constante a través del tiempo, la varianza tampoco debe depender del tiempo, debe ser inmutable, y por último; la covarianza entre los valores de dos períodos contiguos depende del rezago entre éstos y no del período en el cual se calculó la covarianza. Para mayor información véase Pankratz, (1991); Hamilton (1994); Muñoz y Perfetti (2003)

Se escogieron las siguientes variables disponibles y actualizadas en la región: licencias de construcción en el Cauca, ICCV (índice de costos de construcción de vivienda) en Popayán, pasajeros movilizados en Popayán, despacho de cemento en el Cauca, consumo de energía en el Cauca, matrícula de vehículos en Popayán, Sacrificio de ganado en el Cauca y tasa de desempleo en Popayán. 
Gráfico 1. Variación del PIB Cauca y PIB Nacional 2007-2009-I (Base 2000)

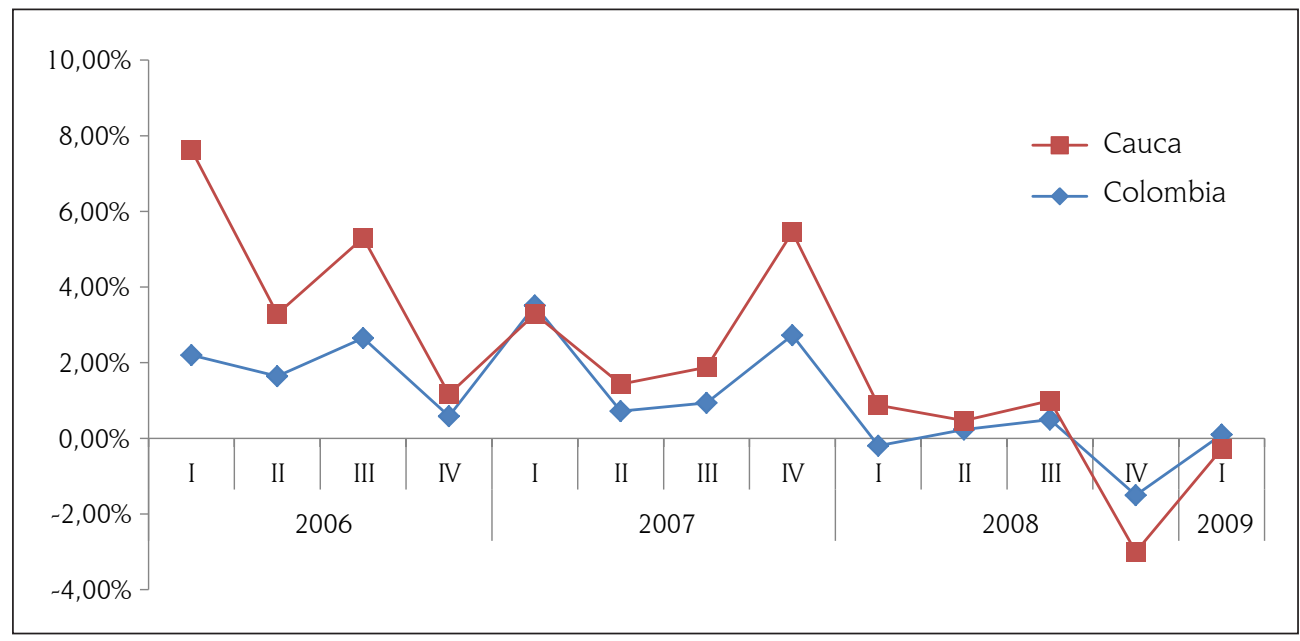

Fuente: elaboración propia. Datos DANE y cálculos propios.

descenso continuo durante el año 2008, a diferencia de los indicadores en la región que evidencian una mejoría a partir del segundo trimestre hasta el cuarto trimestre del 2008. Es decir, mientras la economía nacional se afectaba con la crisis económica y la actividad real disminuía continuamente en el año, el Cauca mostró un comportamiento particular de recuperación de los indicadores líderes y reactivación de la actividad económica real; sin embargo, al final del año, todos los indicadores en la región muestran una caída vertiginosa. Este comportamiento económico puede encontrar explicación en dos aspectos: las medidas de política económica contra cíclicas en la región que hayan minimizado el impacto de la crisis, y el auge de las pirámides en la región, que generó, durante este período, suficiente liquidez para mantener a flote la inversión, el consumo y las actividades de la economía real.

La evidencia muestra que el Gobierno regional no asumió medidas fiscales específicas durante este período que pudiesen contener la crisis, por lo tanto, este comportamiento tiene más explicación en el incremento del consumo y la inversión privada que pudo generarse en los primeros meses de fortalecimiento de las pirámides y el dinero circulante en la economía regional que obtuvieron los "inversionistas" iniciales de las captadoras ilegales.
En este mismo sentido, la caída contundente de varios de los indicadores es coincidente con el derrumbamiento de las pirámides en la región: varios de ellos caen por debajo de los valores nacionales a pesar de haber tenido un comportamiento mejor durante el año. Sin duda, el hecho de que la crisis financiera mundial y el desplome de las pirámides convergieran a finales del 2008 produce gran preocupación frente a lo sucedido en el Cauca, particularmente por los efectos sociales que tendría en términos de incrementos de la tasa de desempleo y los índices de pobreza en año 2009.

\section{EL CONTEXTO SOCIAL EN EL DEPARTAMENTO DEL CAUCA}

Los efectos sociales de la crisis económica y el derrumbe de las pirámides han sido evidentes en la ciudad. Como es sabido, uno de los sectores más afectados con las crisis económicas son la educación y la salud, debido a que en muchas ocasiones se privilegia la búsqueda de trabajo para solventar el gasto de consumo (demanda) familiar sobre los gastos en educación e incluso la misma seguridad social (cotizaciones a salud y pensiones).

En el sector educativo, por ejemplo, se evidencia un incremento del número de estudiantes matriculados en la Universidad del Cauca durante el primer 
Gráfico 2. Matrículas de pre-grado en la Universidad del Cauca. 2006I-2009I

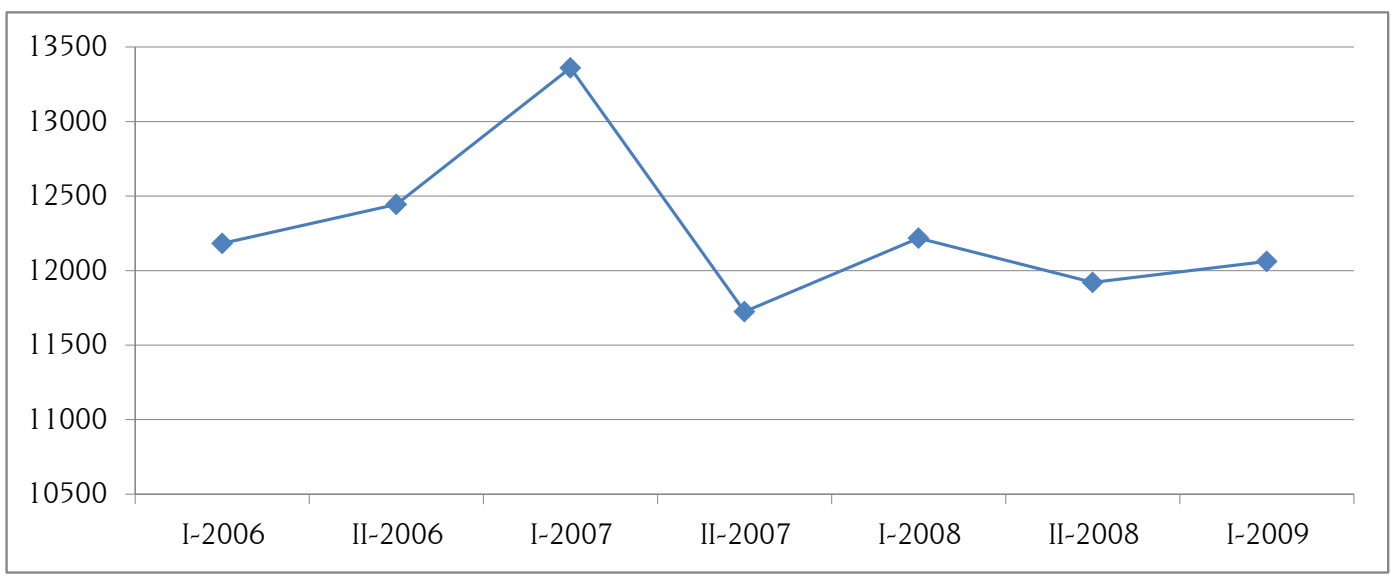

Fuente: elaboración propia. Datos Oficina de Admisiones, Universidad del Cauca.

semestre de 2008, con respecto al último semestre de 2007; sin embargo, el segundo semestre del año, se presenta una disminución de la cantidad de alumnos matriculados en esa institución y una leve recuperación en el primero de 2009. Esto puede tener varias lecturas, una de ellas está relacionada con que en momentos de expansión económica las universidades privadas ganan terreno e incrementan la cantidad de estudiantes matriculados (ver gráfico 2); por el contrario, en épocas de crisis económica la Universidad pública se convierte en una alternativa de educación para los jóvenes.

En términos de los indicadores de salud, si se considera que existe una relación directa entre las variaciones de las afiliaciones al régimen contributivo y nuevos empleos formales, se puede inferir que la crisis económica desatada por el derrumbe de las pirámides dejó sin empleo y, por consiguiente, sin protección, en salud y seguridad social, a miles de familias caucanas, especialmente durante el primer semestre del 2009, como se muestra en la tabla 1.

La tasa de crecimiento total de las afiliaciones al régimen contributivo de salud de los últimos 5 trimestres, a partir del III-2008, muestra un comportamiento cíclico particular. Para los dos trimestres finales de 2008 existe una variación abrupta al pasar en valores totales de $1.42 \%$ a $21.35 \%$. De nuevo, para inicios de 2009 hay un crecimiento bajo frente al anterior período, pero llama la atención la caída tan abrupta en los afiliados a salud que muestra una variación negativa superior al 178\% producto de caídas en $25.84 \%$ para Santander de Quilichao; 26.58\% en Popayán; y 34.10\% en Patía. Finalmente, la situación contraria surge para finales de 2009

Tabla 1. Variación del número afiliados régimen contributivo de salud 2008-2009p*

\begin{tabular}{|c|c|c|c|c|c|c|}
\hline Periodo & Patía & Popayán & Rosas & Santander & Timbío & Total \\
\hline III-2008 & $1,70 \%$ & $0,43 \%$ & $0,00 \%$ & $0,64 \%$ & $-1,35 \%$ & $1,42 \%$ \\
\hline IV-2008 & $4,47 \%$ & $2,84 \%$ & $5,02 \%$ & $3,90 \%$ & $5,12 \%$ & $21,35 \%$ \\
\hline I-2009 & $2,59 \%$ & $-1,09 \%$ & $2,31 \%$ & $0,81 \%$ & $0,87 \%$ & $5,49 \%$ \\
\hline II-2009 & $-34,10 \%$ & $-26,58 \%$ & $-57,92 \%$ & $-25,84 \%$ & $-34,14 \%$ & $-178,58 \%$ \\
\hline III-2009 & $50,23 \%$ & $36,66 \%$ & $79,57 \%$ & $36,77 \%$ & $42,67 \%$ & $245,90 \%$ \\
\hline
\end{tabular}

*NOTA: Total: Agregado de Patía, Popayán, Rosas, Santander de Quilichao y Timbío. Fuente: elaboración propia. Datos Secretaría Departamental de Salud del Cauca. 
donde existe un alto incremento de afiliaciones en los municipios bajo estudio.

Los efectos sociales, particularmente en la seguridad ciudadana, tras el derrumbe de las pirámides se vivieron inmediatamente. De acuerdo con lo establecido por la Policía Nacional Cauca, se presentaron incrementos en delitos como robo a residencias, abigeato y atracos callejeros.

En el primer trimestre de 2009, el hurto a personas y residencias al igual que las lesiones personales alcanzan casi los mismos niveles de todo el año 2008 para el departamento; se inicia un ascenso de estos delitos a partir del IV trimestre de 2008 y mantiene la tendencia creciente durante el primer trimestre de 2009. De esta manera se corrobora la hipótesis que la crisis de las pirámides indujo mayores niveles de inseguridad en Popayán y la región. En cuanto a la proliferación de algunos delitos ya mencionados, como el raponazo, el atraco, el fleteo, la extorsión y el robo de vehículos y residencias, la cifra continúa en aumento. A pesar de que la Policía Nacional asegura que los homicidios en el país han bajado, existe una percepción de inseguridad mucho más alta para la región, en gran parte, producto del impacto recesivo de la crisis de las pirámides, efectos que las autoridades municipales tratan de contrarrestar aplicando medidas preventivas y judiciales contra la delincuencia, entre ellas, la disposición de 2.600 hombres por parte de la Policía Cauca en planes operativos para el fin del año 2009.

Los efectos, pasado un año, continuaban siendo preocupantes también en otros contextos. La Dirección Antinarcóticos de la Policía Nacional, por ejemplo, creó un escuadrón especial para la erradicación del cultivo de Amapola en Nariño y Cauca, debido al incremento en su producción. De acuerdo con estas autoridades, el fenómeno obedecía a la caída de las captadoras ilegales en la región, información respaldada por informes consignados en el diario El País (abril, 2009) y el Tiempo (marzo, 2009). De otro lado, asociaciones agrícolas o ganaderas como Agropemca y Asogan afirmaban que el sector agro de la región suroccidental del país también salió seriamente perjudicado con el fenómeno de las pirámides, debido a que muchos campesinos, productores y cultivadores legales e ilegales, observaron en las pirámides una oportunidad inmejorable de elevar sus ingresos de una forma rápida y fácil.

Finalmente, los damnificados de las pirámides, que fueron en su mayoría personas de los estratos 3, 4 y 5, tras la inversión de los ahorros de toda su vida y de la venta de bienes y propiedades, asisten actualmente a la entrega, por parte del Gobierno

Tabla 2. Hurto por municipios y total Cauca. Anual 2006-2009.

\begin{tabular}{|c|c|c|c|c|c|c|c|c|c|c|c|c|}
\hline \multirow[b]{2}{*}{ Municipio } & \multicolumn{3}{|c|}{2006} & \multicolumn{3}{|c|}{2007} & \multicolumn{3}{|c|}{2008} & \multicolumn{3}{|c|}{2009} \\
\hline & 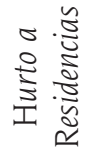 & 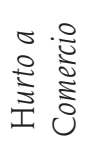 & 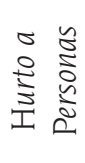 & 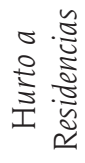 & 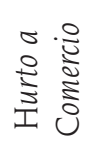 & 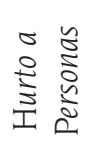 & 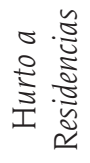 & 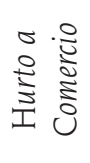 & 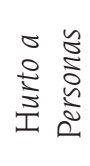 & 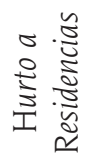 & 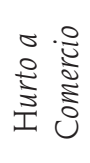 & 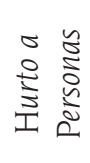 \\
\hline Patía (El Bordo) & 7 & 4 & 13 & 9 & 1 & 32 & 8 & 2 & 13 & & 1 & 8 \\
\hline Popayán (CT) & 203 & 127 & 589 & 205 & 177 & 473 & 106 & 153 & 365 & 57 & 78 & 285 \\
\hline Rosas & & 1 & 3 & & 2 & 5 & & & 1 & & & \\
\hline Santander de Q. & 14 & 9 & 29 & 2 & 8 & 13 & 11 & 11 & 21 & 8 & 6 & 26 \\
\hline Timbío & 6 & 5 & 21 & 4 & 3 & 12 & 1 & 2 & 9 & 1 & 1 & 1 \\
\hline Total Cauca & 230 & 146 & 655 & 220 & 191 & 535 & 126 & 168 & 409 & 66 & 86 & 320 \\
\hline
\end{tabular}

Fuente: Policía Nacional 
nacional a través de entidades como Fiduprevisora y la Superintendencia Financiera, del dinero recuperado en las captadoras, suma que no asciende a los 350 mil pesos por ahorrador, independientemente del capital invertido. Mientras tanto, aún hoy se arguyen todo tipo de explicaciones alrededor de estas captadoras ilegales de dinero y la crisis tras su desplome e intervención, entre ellas una cultura del dinero fácil y del narcotráfico enraizada en nuestra sociedad. Estas reflexiones deben atemperarse con la experiencia en otros países como Albania, donde el valor captado por las pirámides albanesas ascendió a casi la mitad del PIB del país, y con casos no menos graves como los sucedidos hace algunos años en Gran Bretaña, Rusia y Estados Unidos, ante la necesidad de dar una solución efectiva y sostenida a la crisis económica y social que vive la región sur del país.

\section{CONSTRUCCIÓN DE INDICADORES PARA LAS PIRÁMIDES Y LA CRISIS FINANCIERA: METODOLOGÍA ECONOMÉTRICA}

Uno de los objetivos de este trabajo es encontrar los factores que afectaron la economía del Cauca en la coyuntura, con énfasis en lo sucedido durante el año 2008 y el primer trimestre de 2009, especialmente, la relación que existe entre la caída de las pirámides y la crisis financiera internacional con las variables líderes que se consideran como observadoras adelantadas de la actividad económica.

Para ello se construyó un modelo econométrico a partir del diseño de dos indicadores que muestran el comportamiento del impacto de la crisis financiera y las pirámides en la economía regional, así como las correlaciones existentes entre cada uno de ellos con los indicadores líderes de la actividad del sector real de la economía caucana.

\subsection{Metodología para la construcción de los indicadores}

Debido a que muchas variables de orden económico y social se vieron afectadas tanto por la crisis financiera como por las captadoras ilegales de dinero para el Cauca, no es posible seleccionar una de ellas como representativa directa de la situación. Para tal efecto se construyeron dos indicadores compuestos por variables seleccionadas de la actividad económica regional, que muestran el comportamiento de la crisis financiera en la región y el comportamiento de las pirámides, y que intentan recoger con alta precisión el comportamiento de estos dos fenómenos.

\section{VECTORES PROPIOS O EIGENVECTORS}

Tanto para la construcción del indicador de crisis financiera (ICF) como para el indicador de pirámides (IP), se utilizó la metodología de los vectores propios (eigenvectors) ${ }^{3}$. En términos generales, el método intenta "extraer" la información relevante de un conjunto de datos, eliminando de ellos la información redundante, o poco relevante. En términos más precisos, si X es la matriz que relaciona los valores que asumen las variables bajo análisis en cada período de tiempo, se tiene que:

$$
X=\left[\begin{array}{cccc}
x_{11} & x_{12} & \ldots & x_{1 p} \\
x_{21} & x_{22} & \ldots & x_{2 p} \\
\vdots & \vdots & \vdots & \vdots \\
x_{n 1} & x_{n 2} & \ldots & x_{T p}
\end{array}\right]_{T x p}
$$

Donde $\mathrm{X} \in \mathrm{R}^{\mathrm{p}}$, p es el $\mathrm{p}$-ésima variable, y n es el n-ésimo período bajo análisis. El objetivo a partir de este punto, entonces, es lograr transformar la matriz X en otra que se pueda proyectar en $\mathrm{k}$, donde $\mathrm{k}<\mathrm{p}$, es decir, reducir la dimensionalidad de $\mathrm{X}$ desde $\mathrm{p}$ hasta $\mathrm{k}$, y que por tanto, las k-p variables fuesen irrelevantes. Sea F la nueva matriz proveniente de la transformación de la matriz X, los coeficientes de $F$ deben ser ortogonales, es decir, no deben tener ningún tipo de relación lineal, por tanto, no debe existir multicolinealidad. Así, F se puede expresar como:

\footnotetext{
3 Véase, Muñoz y Perfetti (2003), Härdle, W. (1990).
} 


$$
F=\left[\begin{array}{cccc}
F_{11} & F_{12} & \ldots & F_{1 p} \\
F_{21} & F_{22} & \ldots & F_{2 p} \\
\vdots & \vdots & \vdots & \vdots \\
F_{n 1} & F_{n 2} & \ldots & F_{T p}
\end{array}\right]_{T x p}
$$

La técnica de los componentes principales permite reducir la matriz $\mathrm{F}$ hasta un vector columna con la siguiente estructura:

$$
f=\left[\begin{array}{l}
f_{1} \\
f_{2} \\
f_{3} \\
f_{p}
\end{array}\right]_{p x 1}
$$

Para obtener el vector $\mathrm{f}$ se debe tomar la matriz de coeficientes de información de las variables relevantes y descomponerlos en sus vectores de la siguiente forma:

$$
\begin{gathered}
A=\left[\begin{array}{cccc}
a_{11} & a_{12} & \ldots & a_{1 p} \\
a_{21} & a_{22} & \ldots & a_{2 p} \\
\vdots & \vdots & \vdots & \vdots \\
a_{p 1} & a_{p 2} & \ldots & a_{p p}
\end{array}\right]_{p x p} \\
a_{1}=\left[\begin{array}{l}
a_{11} \\
a_{12} \\
a_{13} \\
f_{1 p}
\end{array}\right]_{1 x p} a_{2}=\left[\begin{array}{l}
a_{21} \\
a_{22} \\
a_{23} \\
f_{2 p}
\end{array}\right]_{2 x p} \quad a_{i}=\left[\begin{array}{l}
a_{i 1} \\
a_{i 2} \\
a_{i 3} \\
f_{i p}
\end{array}\right]_{i x p}
\end{gathered}
$$

En notación matricial la matriz $\mathrm{F}$ se puede expresar como:

$$
F_{p x 1}=A_{p x p} X_{p x 1}
$$

Y en notación vectorial, cada componente principal se puede expresar como:

$$
\begin{aligned}
& F_{1}=a_{1}^{T} X_{p x 1} \\
& F_{2}=a_{2}^{T} X_{p x 1} \\
& F_{k}=a_{p}^{T} X_{p x 1}
\end{aligned}
$$

El vector columna formado por estos valores propios en cada caso se constituye en los indicadores que se busca construir.

\section{CICLOS DE LAS SERIES BAJO EL FILTRO HODRICK-PRESCOTT}

A continuación, para determinar el impacto de la crisis y las pirámides en el nivel de actividad económica, los indicadores hallados se relacionan por separado con los indicadores líderes de la economía, propuestos por el DANE, adicionando a éstos los hallados por el Sistema de Información Tecnoeconómica para el Departamento del Cauca (SITEC); al respecto, Gómez, Miler y Rivera (2006, p. 79) argumentan que:

... las variables líderes en el Cauca son cartera financiera nacional, consumo de energía departamental, despacho de cemento departamental, costos de construcción de vivienda departamental, IPC nacional, licencias de construcción Cauca, PIB Cauca, PIB nacional, pasajeros movilizados departamental, sacrificio de ganado departamental, tasa de desempleo Popayán y vehículos matriculados.

Para calcular estos impactos correctamente se debe extraer el componente cíclico, no solo de los indicadores, sino de las variables líderes de la economía regional. Esto se hace necesario porque la información que éstas transmiten es de largo plazo $y$, por tanto, los impactos entre ellas pueden estar sesgados, toda vez que las tendencias ocultan las verdaderas relaciones que subyacen entre ellas. Por tal motivo, se deben transformar tanto los indicadores ICF e IP como los indicadores líderes. En este orden de consideraciones, el componente cíclico se extrajo a través del filtro de Hodrick-Prescott. El ciclo de cualquier serie se define como la diferencia entre el valor de la serie en t y la tendencia en t (en términos logarítmicos). Por tanto:

$$
C_{t}=Y_{t}-T_{t}
$$

En la ecuación anterior no se conoce la tendencia, de tal forma que el filtro de Hodrick-Prescott 
propone para obtenerla la minimización de la siguiente función:

$$
\operatorname{Min} \sum_{t=1}^{n}\left(Y_{t}-T_{t}\right)^{2}+\lambda \sum_{t=2}^{n}\left(\Delta Y_{t}-\Delta T_{t}\right)^{2}
$$

Donde la serie $\mathrm{Y}_{\mathrm{t}}$ es la serie bajo análisis, $\mathrm{T}_{\mathrm{t}}$ es la tendencia, $\left(\mathrm{Y}_{\mathrm{t}}-\mathrm{T}_{\mathrm{t}}\right)$ se conoce como el residuo cíclico, el cual es una estimación de la combinación del componente cíclico y el componente irregular de la serie, y el segundo término es una aproximación de la segunda derivada de la tendencia en el período t. En esta expresión entonces se desea minimizar el error cíclico y la diferencia (derivada) tendencial. De acuerdo con Muñoz y Perfetti (2003, p. 56), "el parámetro 1 es un parámetro de suavización que asume un valor superior a cero, el cual pondera a estas dos expresiones. Este parámetro permite que la tendencia se suavice cuando su valor es alto, y que no lo haga cuando es bajo". Hodrick y Prescott (1980, p. 5) recomiendan $l=14400$ para datos mensuales, $l=1600$ para datos trimestrales, y $\mathrm{l}=100$ para datos anuales. En el presente estudio se siguió la recomendación de $l=1600$, ya que, como se sabe, las series aquí utilizadas tienen una periodicidad trimestral.

\section{MATRIZ DE CORRELACIONES}

Una vez obtenidas todas las variables en ciclos, se obtienen las relaciones entre ellas a través de una matriz de correlaciones simples. El fin del análisis de correlación es medir el grado de asociación lineal entre dos variables medido por el coeficiente de correlación ( $r$ ), tratando las dos variables en forma simétrica, es decir, no hay distinción entre la variable dependiente y la explicativa, lo que sí ocurre cuando se plantea un modelo de regresión.

La correlación puede ser fuerte, débil o nula, y dentro de estas categorías, también se puede establecer si es una relación positiva o negativa. Una de las propiedades del coeficiente de correlación muestral es que puede tener signo positivo o negativo en función de la covarianza muestral de las dos variables. Se encuentra entre -1 y $1(-1 \leq \mathrm{r} \leq 1)$. Es simétrico por naturaleza, es decir, el coeficiente de correlación entre X y Y $\left(\mathrm{r}_{\mathrm{xy}}\right)$ es el mismo que entre Y y $\mathrm{X}\left(\mathrm{r}_{\mathrm{yx}}\right)$. Y por último, aunque es una medida de asociación lineal entre dos variables, esto no implica necesariamente alguna relación causa efecto 4 . Los coeficientes de correlación muestral pueden situarse en una matriz, denominada la Matriz de Correlación R; en el caso de K variables, se tendrá en total $\mathrm{K}(\mathrm{K}-1) / 2$ coeficientes de correlación. Vale la pena decir que el coeficiente de correlación de una variable respecto a ella misma es siempre 1 $\left(r_{11}=r_{22}=\ldots=r_{k k}=1\right)$. La correlación es estadísticamente significativa si su valor absoluto es igual o superior a 0,3 en valor absoluto 5 .

\subsection{Resultados}

\subsection{1 Índice de crisis financiera (ICF) ${ }^{6}$}

El gráfico 3 muestra el comportamiento del indicador crisis financiera (ICF) en ciclo, es decir, en el corto plazo. El indicador fue construido con variables asociadas a la inversión y la producción real.

El índice crisis financiera en el Departamento del Cauca muestra cómo después de un comportamiento solvente de la economía regional en el 2007, a partir del primer semestre de 2008 se inicia un descenso de las variables económicas regionales impactadas por la desaceleración eco-

Para una aplicación de esta metodología véase Gómez, Miller y Rivera (2006, p. 50).

5 Este resultado obedece a que el estadístico de prueba es:

$$
t^{*}=\frac{\rho\left(c_{i}, c_{j}\right) \sqrt{T-2}}{\sqrt{1-\left(\rho\left(c_{i}, c_{j}\right)\right)^{2}}} \sim t_{(T-2)}
$$

Donde $\mathrm{T}$ es el numero de datos, y $\rho(\mathrm{Ci}, \mathrm{Cj})$ es la correlación entre los ciclos bajo análisis.

6 Para la construcción de este índice se seleccionaron los créditos de consumo, cartera neta Colombia, depósitos de cuenta corriente de Cauca, cartera neta de Cauca, créditos comerciales de Cauca, PIB de Cauca, tasa de desempleo de Popayán, inversión neta de Cauca, sacrificio de ganado de Cauca, y finalmente el índice de costos de la construcción de vivienda en Popayán. Cabe anotar que las variables seleccionadas inician para el ICF en el primer trimestre de 2006 debido al creciente desempeño del sector financiero para este año. Además, las variables están expresadas en logaritmos para reducir su varianza. 
nómica mundial y nacional. Los canales mediante los cuales una crisis internacional se transmite a las economías emergentes pueden ser de carácter real o financiero. En el primer grupo se encuentran el debilitamiento de las exportaciones, la caída de las remesas y la pérdida de confianza de consumidores e inversionistas. En el segundo informe al Congreso realizado por el Banco de la República (2008, p. 23) manifiesta que "también debe tenerse en cuenta la reducción de los flujos de capital y el difícil acceso a recursos de crédito externo".
La crisis financiera internacional impactó el crecimiento económico regional a través de las variables de la economía real. De manera particular, este crecimiento ha estado fuertemente jalonado en los últimos años por el sector industrial, que ha superado el 20\% de participación en el PIB regional. La crisis financiera mundial afectó dramáticamente este sector en el país; de acuerdo con el DANE, en los primeros 10 meses del 2008, la producción del sector manufacturero cayó 1,86\%, y en octubre, la producción registró una estruendosa caída de

Gráfico 3. Índice de corto plazo crisis financiera (CICF). Trimestral 2006-2009.

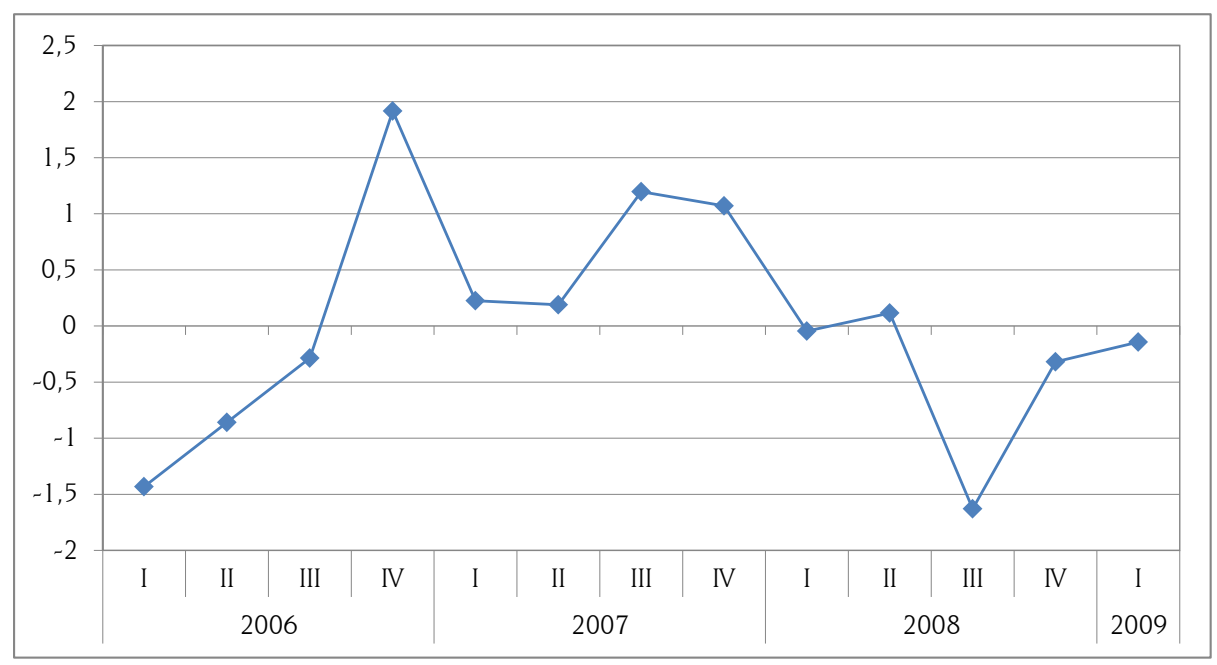

Fuente: elaboración propia

Gráfico 4. Índice de corto plazo pirámides Cauca (CIP). Trimestral 2006-2009.

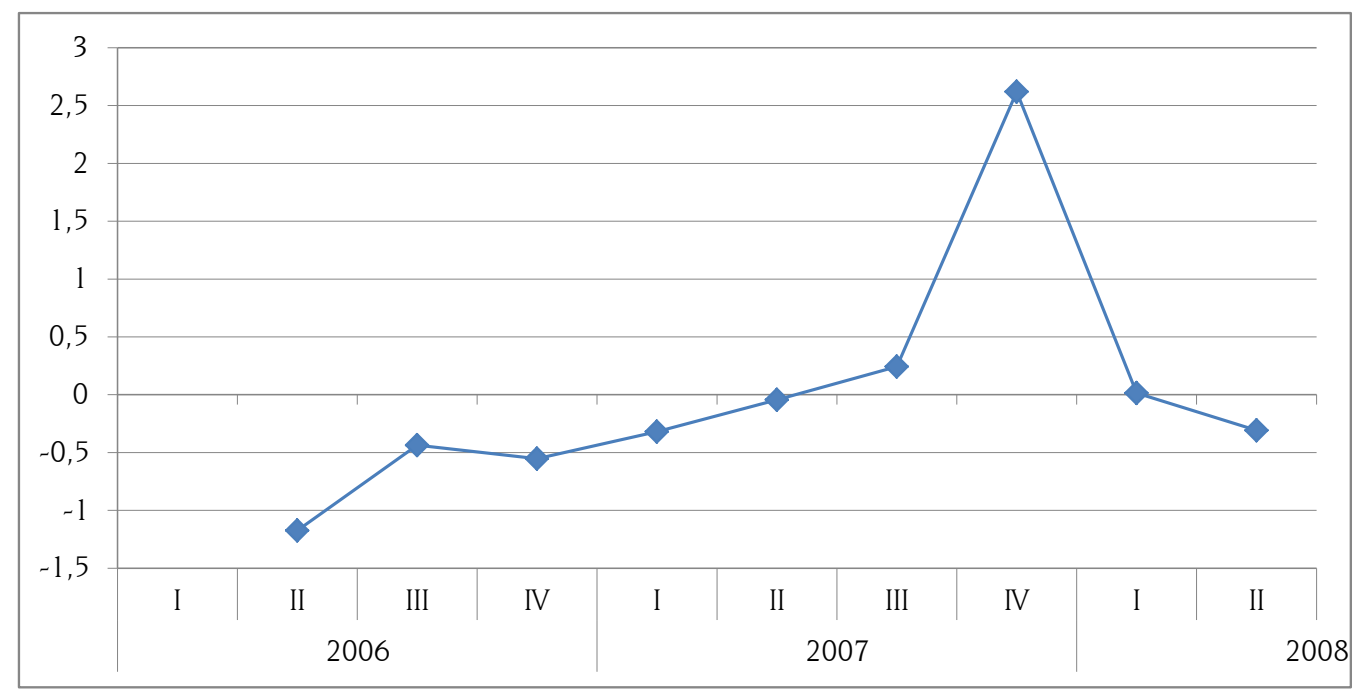

Fuente: elaboración propia 
7,4\%. Las ventas registraron un descenso de 5,50\%, mientras que el empleo en el sector cayó 3,73\%. Con este panorama, el sector manufacturero caucano también fue impactado por la crisis y generó efectos de desaceleración de la economía regional. Otro sector importante que aporta al PIB Regional, es el agropecuario; sin embargo, según CPC (2009), "el sector agropecuario colombiano no atraviesa por el mejor momento pues ha crecido menos que la economía en su conjunto durante las últimas tres décadas". Esto se debe a varios factores como la falta de fuerza laboral calificada, la baja inversión en investigación y desarrollo en el sector agropecuario y otros aspectos de carácter estructural como la inequitativa tenencia de la tierra o la permanencia del conflicto armado en las zonas rurales. En el caso del sector comercio, la situación en el país no ha sido nada halagadora y en el suroccidente, según Fenalco (2009) "la contracción se agudizó más a finales del 2008", no sólo como consecuencia de la desaceleración económica generalizada en todo el país sino por el efecto de la caída de las pirámides; los cálculos de Fenalco (2009) sugieren que "mientras para el agregado nacional las ventas crecieron $0.5 \%$, en Nariño, Cauca y Putumayo se registró una disminución del orden del 6\% en términos reales".

El punto más profundo del coletazo de la crisis en el Departamento se dio en el tercer trimestre del 2008; a partir de ese momento se inicia una recuperación que, en todo caso, no alcanza a generar crecimientos positivos hasta el primer trimestre de 2009.

\subsection{2 Índice pirámides (IP) ${ }^{7}$}

El gráfico 4 muestra el comportamiento del indicador pirámides en el ciclo, es decir, en el corto

7 En cuanto a éste índice, las variables incluidas fueron créditos de vivienda de Cauca, créditos de consumo de Cauca, microcréditos de Cauca, CDT de Cauca, depósitos de ahorro de Cauca, tasa de desempleo de Popayán, consumo de energía de Cauca, giros de Cauca, industria y comercio de Popayán e impuesto predial de Popayán. Cabe señalar que el IP inicia en el mismo trimestre pero del año 2007, debido a que en este período aparecen en la región las primeras captadoras, además, las variables están expresadas en logaritmos para reducir su varianza. plazo. Vale recordar que este indicador fue construido con variables económicas fundamentadas en el consumo. El indicador inicia en el 2007, debido a que es en ese momento en el cual ingresa al Cauca la pirámide DMG.

El IP inicia en el 2007 con un incremento sostenido y a partir del segundo trimestre del 2008 muestra un crecimiento rápido y formidable. El punto máximo de crecimiento del indicador se encuentra en el tercer trimestre del 2008. Esta circunstancia coincide con el auge que se vivió en la región con las captadoras ilegales de dinero, principalmente DMG y DRFE, aunque en ese período surgieron otras más pequeñas y menos reconocidas que cerraron sus puertas rápidamente huyendo con los dineros de los "inversionistas". El desplome se evidencia en el último trimestre de 2008 y la situación de los indicadores económicos afectados por las pirámides empeora el diciembre de 2008 y el primer trimestre de 2009.

Con modalidades un poco distintas, estas dos pirámides (DMG, DRFE) ingresaron al Cauca atrayendo "inversores" sobre la promesa de altos retornos. En cuestión de meses, el fenómeno se había dispersado por toda la región. DRFE abrió oficinas en municipios como Patía, Rosas, Timbío, Santander de Quilichao y Popayán, en donde se agolpaban personas de todo el Departamento para depositar sus ahorros que serían devueltos un mes más tarde con una utilidad entre el 70\% y 150\%.

Indiscutiblemente, mucho dinero fue puesto en circulación en ese momento. Llama la atención, por ejemplo, el comportamiento de algunas variables de la economía regional como el crédito de consumo, pues mientras en el país la Asobancaria (2008, p. 8) indicaba que la reducción de los desembolsos para los créditos de consumo era significativa en el primer semestre de 2008, como efecto del incremento en las tasas de interés y la mayor precaución de los hogares a la hora de endeudarse, en el Departamento se registraba un incremento de los créditos de consumo en el mismo período. 
Gráfico 5. Variaciones trimestrales del crédito de consumo Cauca 2006-2009. Precios constantes base 2000.

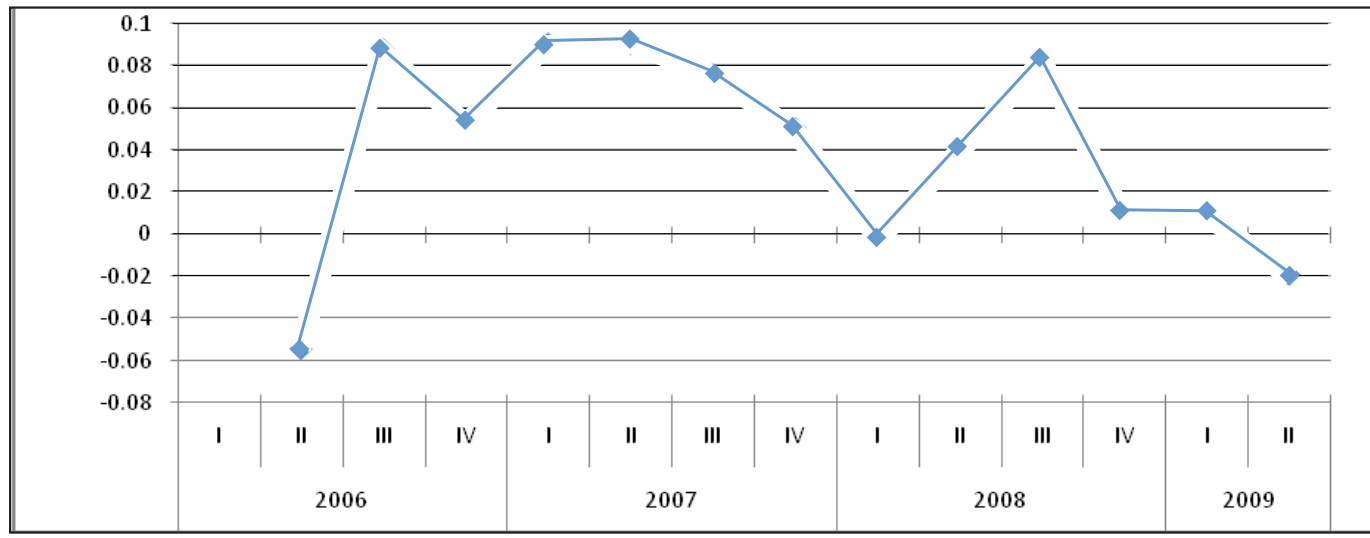

Fuente: elaboración propia. Datos Superfinanciera.

Gráfico 6. Captaciones del sistema financiero Cauca. Trimestral 2005-2009.

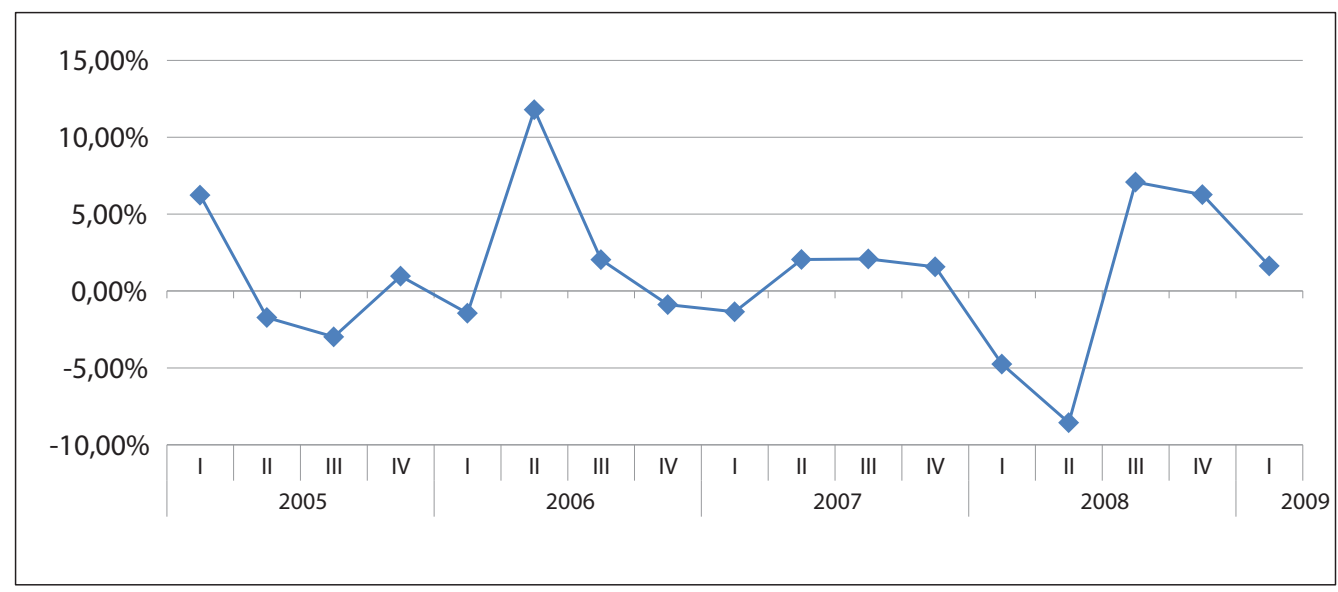

Fuente: elaboración propia. Datos Superfinanciera

Gráfico 7. Variación giros en el Cauca. Trimestral 2006-2009.

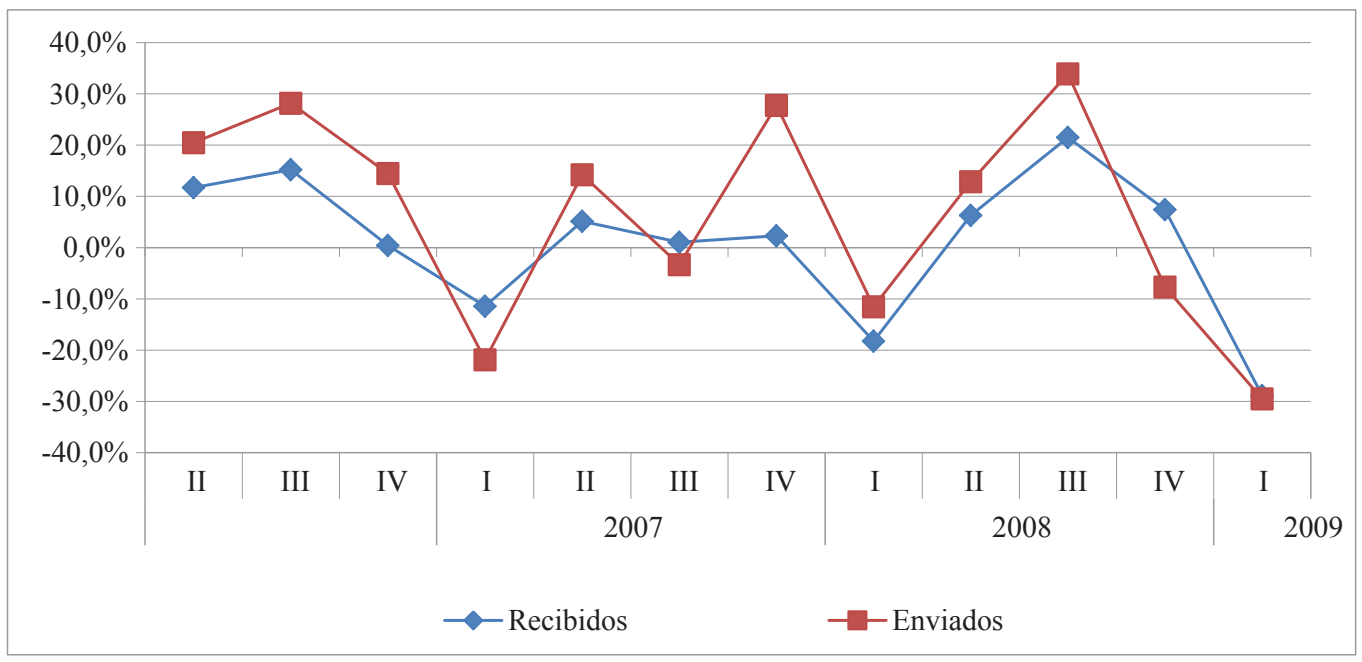

Fuente: elaboración Propia. Datos Cámara de Comercio del Cauca 
De otro lado, las captaciones de dinero en el interior del sistema financiero tuvieron un descenso vertiginoso en el primer semestre de 2008, con un decrecimiento de $8.56 \%$ a final del segundo trimestre, donde inicia una recuperación pero vuelve a caer simultáneamente con el derrumbe de las pirámides.

Una hipótesis que explicaría el comportamiento de estas dos variables radica en la decisión del público de "invertir" en las pirámides, de manera más acentuada entre el segundo y cuarto trimestre de 2008, disminuyendo los depósitos de los ahorradores en el sistema financiero y, por otra parte, solicitando créditos de consumo (más expeditos debido a que tienen menos trámites y requerimientos) que serían "invertidos" en las captadoras ilegales de dinero con el fin de obtener altas tasa de rentabilidad. De esta manera se podría explicar el comportamiento casi contra cíclico de estas dos variables fundamentales del sistema financiero en el escenario regional y nacional.

La liquidez generada en la economía regional durante los meses de auge de las pirámides debió de tener impactos positivos sobre el consumo entre el segundo y cuarto trimestres. Los giros de dinero intra regionales y el movimiento de las empresas de giros en la región así lo muestran.

La escasa presencia bancaria en los municipios diferentes a Popayán ha hecho que empresas dedicadas a realizar giros de dinero brinden a los usuarios la posibilidad de enviar y recibir dinero proveniente de distintos lugares del Departamento, seguros y a bajo costo. El comportamiento de los giros tanto recibidos como enviados no tiene una tendencia clara. Las variaciones que se presentan de un trimestre a otro son bastante importantes, especialmente durante la coyuntura de las captadoras ilegales. El gráfico 7 muestra dos picos importantes en los giros enviados por los caucanos; el primero en el último trimestre de 2007, que coincide con la expansión económica generada por el alto crecimiento de la economía durante ese año, y el segundo, el tercer trimestre del 2008, período que se correlaciona con el auge de las pirámides.

Nuevamente se evidencia, que durante el segundo y tercer trimestre de 2008, a pesar de la recesión económica que se vivía en el país, en el Cauca se presentaba un exceso de liquidez en la economía, producto de los dineros que obtuvieron los primeros "inversionistas" de las pirámides. La caída de las captadoras ilegales repercutió fuertemente en los giros enviados y recibidos; esto puede observarse en el cuarto trimestre de 2008, ya que presenta una significativa disminución en los mismos, crisis que se agudiza en el 2009, pues se llega a las tasas más bajas en los últimos dos años.

El consumo de los hogares representa aproximadamente el $66 \%$ del gasto; por lo tanto, su contribución al crecimiento o desaceleración del PIB es importante. El fenómeno de las pirámides estuvo relacionado de manera más directa con variables de consumo, por ello a pesar de la disminución del consumo en el orden nacional durante el 2008 (-0.5\%), en el segundo y tercer trimestre éste no disminuyó sustancialmente en la región. Por vía contraria el desplome de las pirámides debió impactar negativamente las variables relacionadas con el consumo. En efecto, los picos más altos de pago del predial se dan para el segundo trimestre de 2007 y primer trimestre de 2008, con variaciones de $439,20 \%$ y 509,33\%, respectivamente; esta circunstancia obedece a los incentivos ofrecidos por la alcaldía municipal, para el recaudo en el primer trimestre del año.

A partir del segundo trimestre de 2008 hasta el segundo trimestre de 2009, el pago de impuesto predial presenta variaciones negativas; sin embargo, llama la atención lo sucedido entre el segundo y el cuarto trimestre de 2008, en el que se observa un leve crecimiento del recaudo; esto coincide con lo expresado anteriormente: las condiciones del consumo de los hogares mejoraron durante el II y IV trimestres de 2008, momento en el que las pirámides inyectaron dinero a la economía. 
Gráfico 8. Índice crisis financiera (CICF) e índice pirámides (CIP). Trimestral 2006-2009.

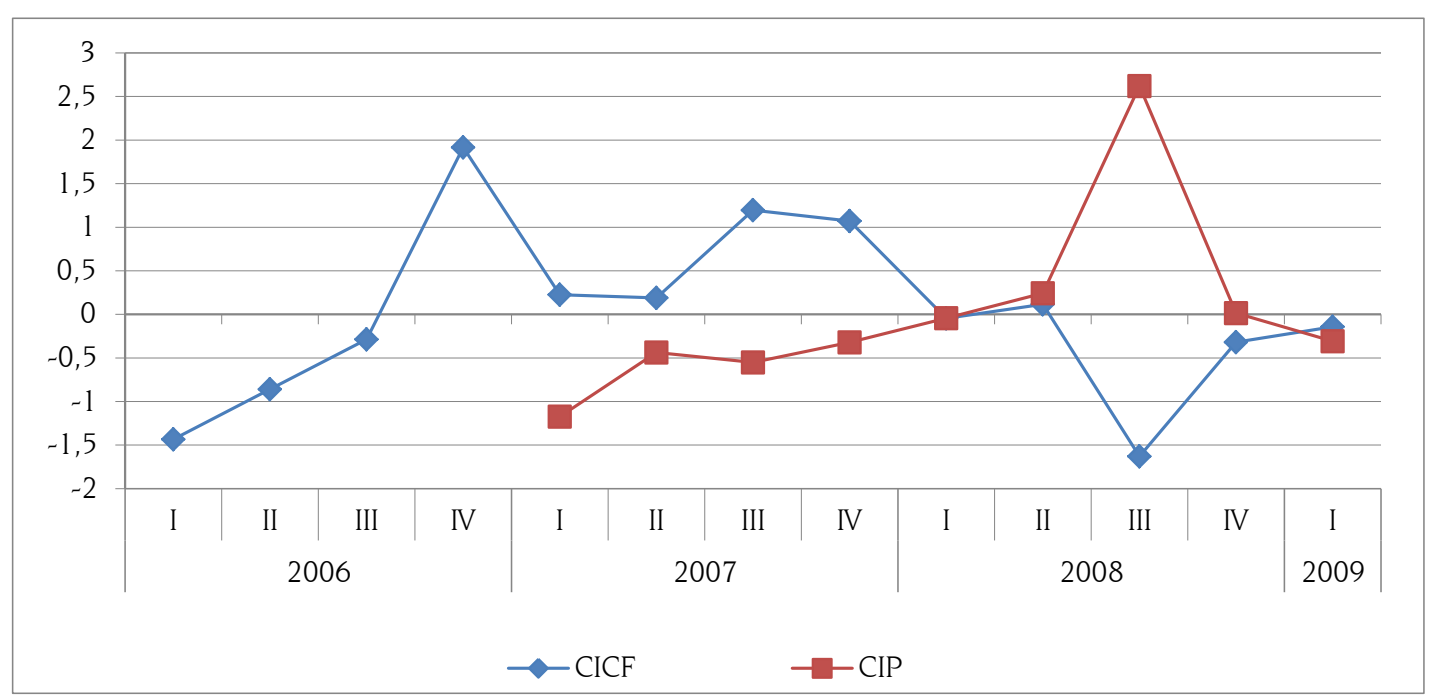

Fuente: elaboración propia

Tabla 3. Correlaciones entre los indicadores ICP e IP y las variables líderes

\begin{tabular}{|l|c|c|}
\hline \multicolumn{1}{|c|}{ Variables $^{9}$} & CICF & CIP \\
\hline CICF & 1,00 & $-0,79$ \\
\hline CIP & $-0,79$ & 1,00 \\
\hline C_LCCOL & 0,58 & $-0,29$ \\
\hline C_LCE & 0,38 & $-0,47$ \\
\hline C_LDC & 0,21 & $-0,22$ \\
\hline C_LICCV & $-0,23$ & 0,31 \\
\hline C_LIPC & $-0,73$ & 0,45 \\
\hline C_LLC & 0,12 & 0,11 \\
\hline C_LPIBC & 0,12 & 0,30 \\
\hline C_LPIBN & 0,22 & $-0,13$ \\
\hline C_LPM & 0,69 & $-0,38$ \\
\hline C_LSG & 0,00 & 0,43 \\
\hline C_LTD & $-0,11$ & $-0,46$ \\
\hline C_LVM & 0,61 & $-0,22$ \\
\hline
\end{tabular}

Fuente: elaboración propia a partir de Gómez, Miler y Rivera (2006).

En negrilla se encuentran los valores estadísticamente significativos, pues superan en valor absoluto a 0.30; es decir, la variable que guarda correlaciones con cada uno de los índices. La letra " $C$ " que antecede a las variables significa que se encuentran en ciclo, y la primera letra "L", que son en logaritmos naturales.

2 ICF: índice de crisis financiera, IP: índice de pirámides, LCCOL: logaritmo natural de la cartera Colombia, LCCOL: logaritmo natural del consumo de energía, LCE: logaritmo natural de despacho de cemento Cauca, LICCV: logaritmo natural del índice de costos de construcción de vivienda, LIPC: logaritmo natural del IPC nacional, LLC: logaritmo natural de licencias de construcción, LPIBC: logaritmo natural del PIB Cauca, LPIBN: logaritmo natural del PIB nacional, LPM: logaritmo natural de pasajeros movilizados, LSG: logaritmo natural sacrificio ganado, LTD: logaritmo natural de la tasa de desempleo Popayán, LVM: logaritmo natural de vehículos motorizados. 
Gráfico 9. Correlación PIB Cauca (C_ _ LPIBC) y el indicador crisis financiera de largo plazo (C _ LICP). Trimestral 2006-2009.

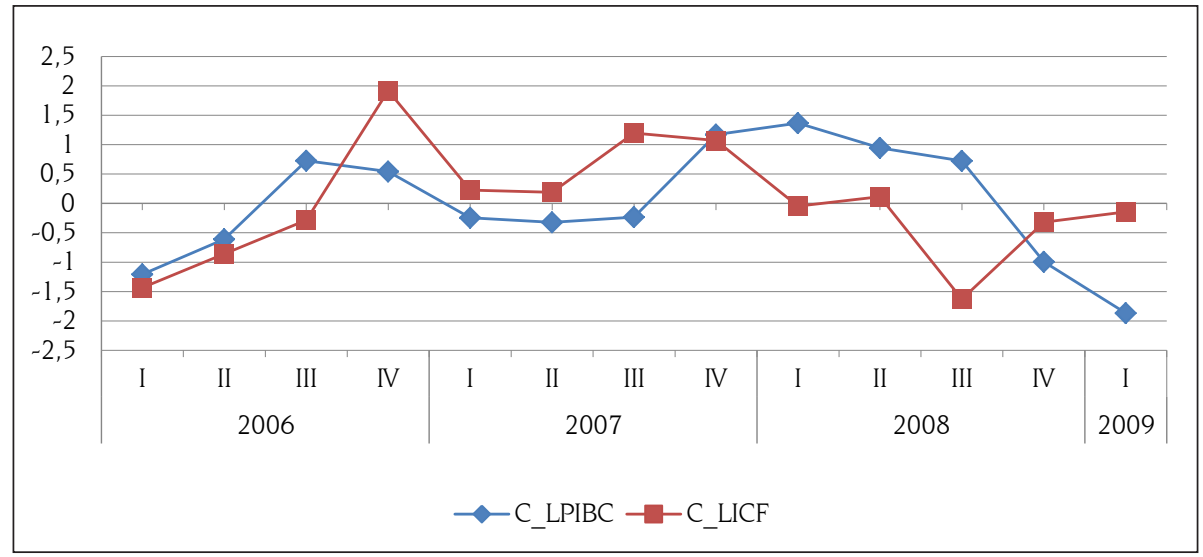

Fuente: elaboración propia.

\subsection{Comparación de índices en el corto plazo}

Extrayendo las correlaciones simples entre ambos ciclos, se muestra que ésta es alta y negativa (-0.79), lo que evidencia una relación contra cíclica y bastante elevada, además de estadísticamente significativa. En otras palabras, en el mismo período, las dos variables exhiben comportamientos contrarios. Esto indica que mientras la región estaba siendo impactada negativamente por la crisis financiera mundial y el indicador construido para el Departamento (ICF) tenía una tendencia decreciente con su punto más bajo en el tercer trimestre del 2008, el indicador pirámides (IP) tenía una tendencia creciente y una cúspide en el mismo período.

El efecto de este comportamiento para la economía del Cauca radica en que las pirámides y la liquidez temporal que produjeron en la economía regional generaron un soporte económico frente a la crisis financiera internacional que impactaba al país y a la región.

\subsubsection{Correlaciones entre variables}

La tabla 3 contiene las correlaciones entre las variables líderes contenidas en Gómez, Miler y Rivera (2006) y los índices construidos en este estudio ${ }^{8}$. Como se planteó anteriormente, uno de

8 Los indicadores líderes, de acuerdo con el Sistema de Información Tecno-económica (SITEC, 2006) son las licencias los hallazgos fundamentales de este estudio está en que las pirámides y la liquidez temporal que produjeron en la economía regional generaron un soporte económico frente a la crisis financiera internacional que impactaba al país y a la región. Esta afirmación puede ser sustentada a partir del análisis de la matriz de correlaciones que muestra que la crisis financiera no tiene una alta correlación con el PIB del Cauca, a diferencia de la correlación alta y positiva del PIB regional con el indicador pirámides. Es decir, en el comportamiento del PIB regional en el 2008, tuvo mayor incidencia lo sucedido con las pirámides que lo sucedido con la crisis financiera mundial.

Si se compara el ciclo regional con el indicador de pirámides, gráfico 10 , se evidencia que en el último período de 2008 y el primer trimestre del 2009 fueron difíciles para la economía regional, pues unida al impacto de la crisis financiera sobre la economía, el derrumbe de las pirámides en el Cauca agudizó la desaceleración económica.

Mientras en el ámbito nacional, el PIB nacional respondía a las medidas contra-cíclicas asumidas por el Banco de la República y el Gobierno, en la

de construcción del Cauca, ICCV de Popayán, Pasajeros movilizados de Popayán, Despachos de cemento en Cauca, Consumo de energía en Cauca, Sacrificio de ganado en Cauca. Las variables que se adelantan al PIB regional son: la tasa de desempleo de Popayán, PIB nacional y el IPC. 
región, el PIB se iba a pique: entre el tercer trimestre de 2008 y el primero de 2009 el PIB Nacional presentó un crecimiento de 0,10\%, mientras el PIB del Cauca presentó un crecimiento negativo de -0,38\%.

\subsubsection{Otras correlaciones}

Por otro lado, el IPC nacional guarda una correlación significativa con ambos índices; la inflación mundial y nacional en el 2007 presenta un incremento escalonado, con el aumento en el precio de los alimentos y de los hidrocarburos, que poco a poco causaron el encarecimiento de los demás bienes de la economía. En cuanto al índice pirámides, su impacto sobre los precios fue causado por la cantidad de dinero circulante en la economía local, el cual se tradujo fundamentalmente en consumo. La variación que ha presentado el IPC nacional desde el primer trimestre de 2008 hasta el primero de 2009, ha evidenciado ritmos decrecientes, pero no ha habido una disminución significativa de los precios. Sin embargo, el tercer trimestre de 2009 se caracterizó por una inflación decreciente en niveles cercanos al punto medio de la meta de largo plazo, y con expectativas de inflación en descenso.

En cuanto a la movilización de pasajeros, existe una alta correlación entre los pasajeros movilizados y los índices crisis financiera y pirámides. Una disminución en la cantidad de dinero merma el ingreso que se destina a bienes y servicios no necesarios (comidas fuera del hogar, gastos en diversión, esparcimiento y turismo); la movilización y el desplazamiento de pasajeros muestra una disminución significativa (-3.73\%) en el último trimestre de 2008 (siempre tiene una tendencia positiva por ser época de vacaciones de fin de año), lo que evidenció la falta de liquidez de la economía caucana en este período, producto de la crisis financiera y de la caída de las pirámides. De la misma manera, el incremento significativo de los pasajeros movilizados para el tercer trimestre del año se explica por la alta correlación existente entre las pirámides y esta variable, ya que en este período se reconoce el auge de las captadoras ilegales.

La correlación entre la crisis económica y la variable desempleo en Popayán no es significativa, mientras que la existente entre éstas y las pirámides sí lo es. Este comportamiento explica las razones por las cuales, a pesar del impacto de la crisis financiera en la región, la tasa de desempleo tuvo una tendencia decreciente durante el año; sin embargo, en el último trimestre de 2008 (derrumbe de las pirámides) el desempleo se incrementa, aun en una época en la que históricamente el desempleo desciende por

Gráfico 10. Correlación PIB Cauca e indicador pirámides

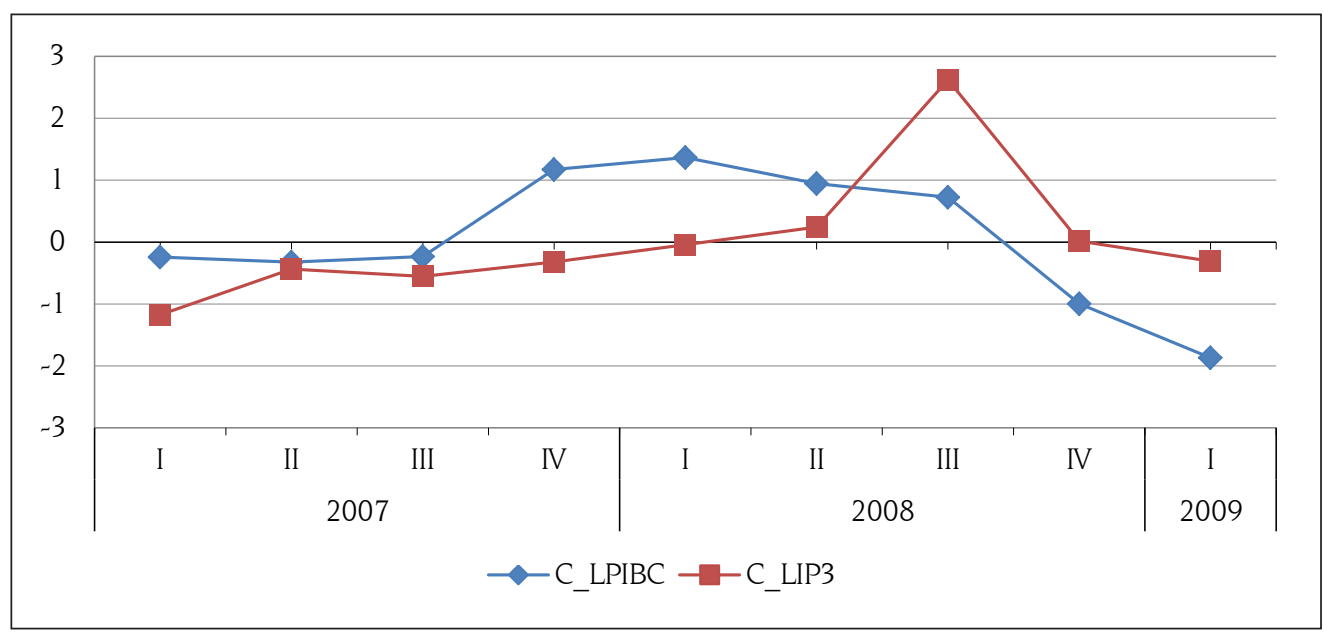

Fuente: elaboración propia. 
ser temporada navideña. Esta circunstancia ratifica el impacto que tuvieron las pirámides sobre el sector formal de la economía regional

En lo que respecta al consumo de energía eléctrica en el Departamento, la alta correlación que muestra esta variable con los dos indicadores, tanto el de crisis financiera como el de pirámides, permite observar un comportamiento bastante interesante. En los dos primeros trimestres de 2008 el consumo de energía disminuye en más de dos puntos porcentuales de un trimestre al otro (2.60\%), resultado de la austeridad provocada por la crisis financiera internacional, sin embargo, para el tercer trimestre de 2008 el consumo de energía eléctrica aumenta en más de tres puntos porcentuales; este comportamiento se explica por las expectativas de alto consumo que se generaron, pues este período coincide con el auge de las pirámides. Como en todos los indicadores anteriores, el fin de año muestra un retroceso en el crecimiento del indicador.

\subsection{Montos de inversión y perfil de los "inversionistas"}

La Superintendencia de Sociedades de Colombia realizó un censo con las personas que se encontraban inscritas en las diferentes bases de datos de las pirámides y/o que poseían recibos que confirmaran los montos de dinero invertidos y la propiedad sobre los mismos, de tal manera que se pudiera decidir con mayor facilidad, si debería o no devolverse dinero. La base de datos obtenida incluye principalmente información de los "inversionistas" de DRFE en los departamentos de Nariño y Cauca; no se contó con información suficiente para depurar la base de datos que permitiera conocer con precisión las cifras del departamento del Cauca.

El censo de la Superintendencia arrojó un total de 192.116 personas en la región que estuvieron directamente involucradas con las pirámides; 151.532 pertenecen al grupo de personas aprobadas, es decir, quienes "invirtieron" un monto específico de dinero y que no les fue devuelto parcial o totalmente. Las 40.584 personas restantes están catalogadas en el grupo de rechazados; personas que al invertir un monto determinado de dinero recibieron la totalidad del mismo o un monto mayor al inicialmente colocado en la pirámide. Cabe considerar que fuera de las 192.116 personas involucradas, existen 152.888 personas más consideradas como condicionadas, es decir, de quienes aún no se tiene claro si invirtieron o no y en caso de haberlo hecho, si recibieron o no dinero.

Según la base de datos de la Superintendencia, la pirámide DRFE recaudó alrededor de $\$ 2,909,423,575,924.70$, de los cuales $\$ 2,295,348,393,514.87$ alcanzaron a devolverse. El

Gráfico 11. Monto de inversiones DRFE distribuido por género

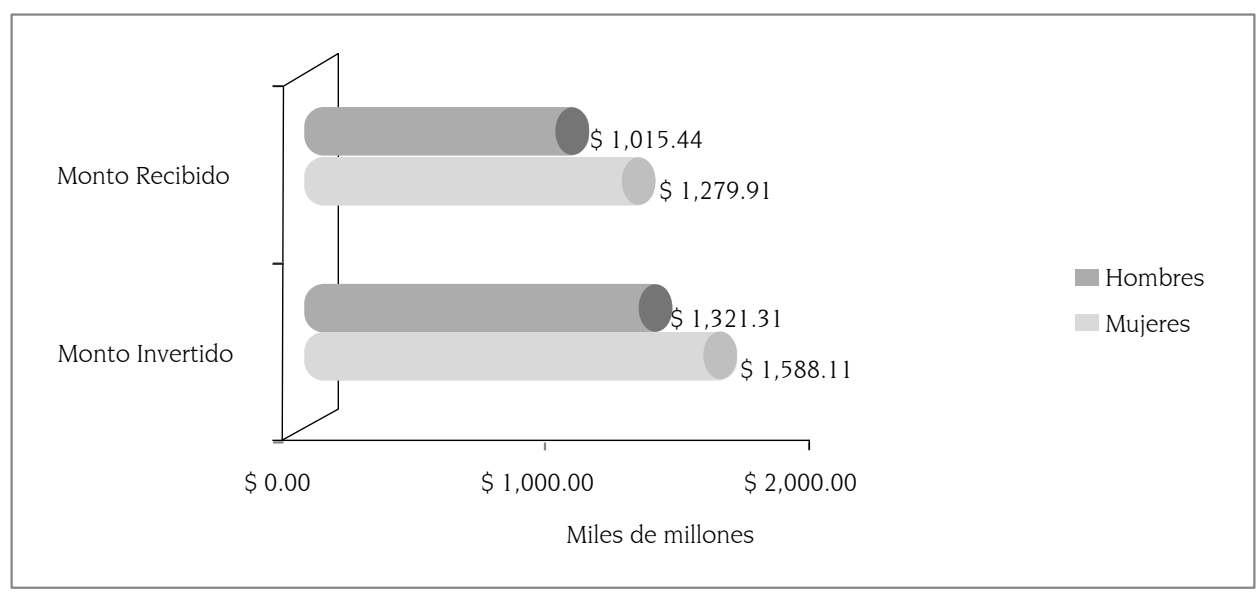

Fuente: elaboración propia. Datos Superintendencia de Sociedades de Colombia. 
$80 \%$ del valor devuelto fue entregado como ganancia de los que inicialmente invirtieron.

\subsubsection{El perfil de los "inversionistas"}

Del total de personas que decidieron participar en las pirámides, las mujeres invirtieron $\$ 1,588,110,538,161.40$, y recibieron $\$ 1,279,911,538,981.07$ antes de la caída de las mismas. Los hombres, por su parte, invirtieron $\$ 1,321,313,037,763.30$, y recibieron $\$ 1,015,436,854,533.80$ (gráfico 14).

De acuerdo con la base de datos, las mujeres se convirtieron en las mayores "inversionistas" de DRFE (53.16\%) y obtuvieron, en este mismo sentido, las mayores pérdidas, es decir, \$308.198.999.180 de pesos $^{9}$. Las mujeres por su parte, representan un 46.84\% de los "inversionistas" de DRFE y obtuvieron pérdidas de $\$ 305.876 .183 .230$ de pesos.

Gráfico 12. Población femenina y masculina inversionista en DRFE 2008. Total regional.

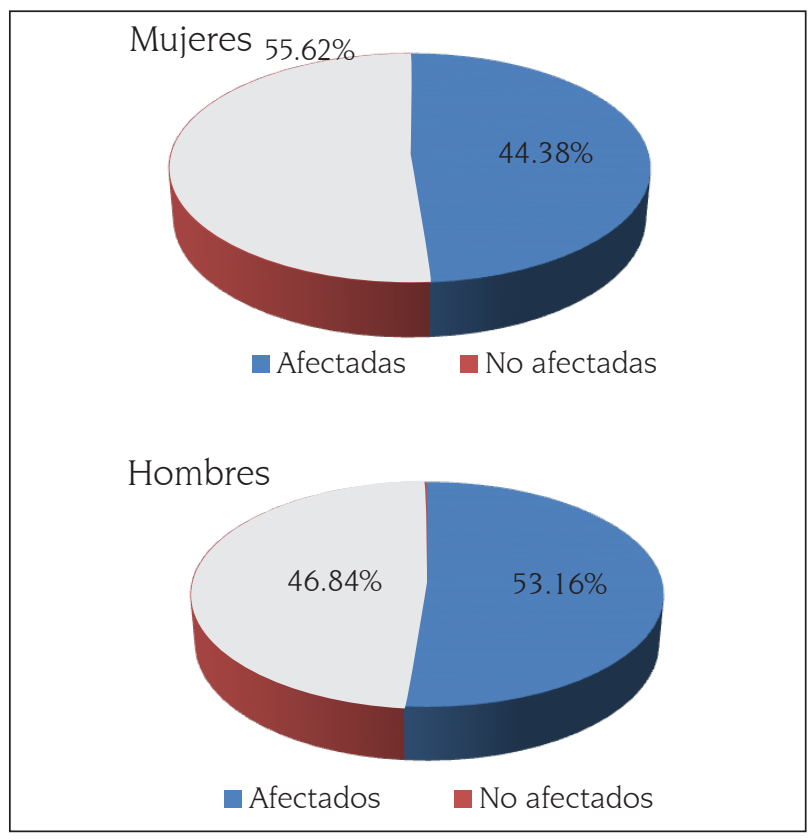

Fuente: elaboración propia. Datos: Superintendencia de Sociedades de Colombia

9 Llama la atención que el mayor monto de inversión reportado en la Base de Datos de la Superintendencia, lo realizó una mujer que invirtió aproximadamente \$2'507.754.674, alcanzó a recibir \$418.550.800 y perdió \$2’089.203.874 con la caída de las pirámides.
La decisión de las mujeres de invertir en las pirámides puede estar asociada a diversos factores relacionados con los buenos hábitos de ahorro que las caracterizan, con la circunstancia de que hoy, como lo dice Cánovas (2008, p. 2) "cada vez más mujeres deben ponerse al frente del sustento económico de sus familias, las mujeres son más abiertas en sus estrategias de inversión y más atentas a escuchar cosas nuevas o ideas diferentes a sus opiniones".

En todo caso, los efectos de la crisis económica y social desatada por el derrumbe de las pirámides afectará de manera diferenciada a hombres y mujeres; los cambios que se generen en los trabajos y en la vida en general de las mujeres y hombres dependerán mucho de la evolución del desempleo. De acuerdo con Larrañaga (2009. p 116):

... si el desempleo continúa afectando más a sectores como el de la construcción o la industria habrá más hombres desempleados y aumentará el número de familias que dependan económicamente en exclusiva de las mujeres, evidentemente esto deteriorará la economía de muchas familias que no sólo pasan de depender de dos sueldos a depender de uno solo, sino que, en general, pasarán a depender del menor de los dos salarios porque como es sabido, y los datos en este sentido parecen variar muy poco con los años, los salarios de las mujeres son en general inferiores a los de los hombres.

En el caso de que el desempleo afecte tanto a las mujeres como a los hombres, algo bastante probable ahora que el desempleo ha empezado a afectar mucho al sector servicios en Popayán donde se emplean más del 80\% de las mujeres, conviene recordar que la situación de las mujeres en desempleo es peor que la de los hombres, pues están menos protegidas y tienen menos acceso a recursos. Otra consecuencia de la crisis, muy ligada al aumento del desempleo, es una mayor precarización y probablemente un empeoramiento de las condiciones laborales de muchas personas, particularmente de las mujeres. Finalmente está claro que la crisis 
afecta no sólo el trabajo remunerado sino también el no remunerado porque el trabajo no mercantil tiene un carácter contra cíclico y normalmente suele adaptarse a la situación económica. Así, en épocas de bonanza económica se agudiza la tendencia a la mercantilización de algunos trabajos domésticos y en épocas de crisis sucede lo contrario. Al respecto Larrañaga (2009, p. 118) señala que:

... en situaciones de desempleo y disminución de la renta el bienestar de las familias se deteriora menos de lo que reflejan los datos económicos a costa de una mayor cantidad de trabajo doméstico y de cuidados y este aumento de la carga de trabajo no remunerado recae en su inmensa mayoría sobre las mujeres.

\subsubsection{Inversionistas según código CIIU}

Entre los más de 100 mil afectados por la caída de las pirámides en el Cauca se cuentan, además, comerciantes, empleados y demás personas vinculadas con alguna labor del sector agropecuario. Toda la ciudad se afectó en algún modo, desde los centros comerciales, pasando por taxistas, cooperativas de empleados, medios de comunicación, profesores, distribuidores mayoristas, plazas de mercado, bares, y discotecas.

Cruzando las bases de datos de la Superintendencia de Sociedades de Colombia y la de comerciantes inscritos en la Cámara de Comercio del Cauca (CCC), se puede observar que el monto total invertido por personas que están relacionadas directamente con algún sector económico formal en el Cauca es de \$22'403.207.119 millones de pesos, de los cuales $\$ 12^{\prime} 883.091 .202$ millones de pesos fueron devueltos. No obstante, con la caída de las pirámides, se estima que las pérdidas que afrontaron las personas que están legalmente constituidas en algún sector económico son de \$9'545.427.777 millones de pesos, aproximadamente el $42.61 \%$ de lo que se recibió.

Los sectores más afectados, según el código CIIU, fueron el de comercio al por mayor y al por menor, reparación de vehículos automotores, motocicletas, efectos personales y en seres domésticos, pues el 63.16\% de las personas que se encuentran registradas en la Cámara de Comercio del Cauca en este sector tomaron la iniciativa de invertir en las pirámides (gráfico 13). Se estima que estos comerciantes invirtieron \$14'338.500.621 millones de pesos aproximadamente, de los cuales se alcanzaron a recibir \$8'161.086.044 millones de pesos como devolución de lo invertido. El sector comercio afronta pérdidas por este fenómeno de aproximadamente $\$ 6^{\prime} 146.200 .077$ millones de pesos. Los comerciantes más afectados son los de la ciudad de Popayán, pues sus pérdidas ascendieron a \$2'090.928.651 millones de pesos. El segundo sector más afectado por la caída de las pirámides es

Gráfico 13. Inversionistas DRFE según código CIIU. Total Cauca.

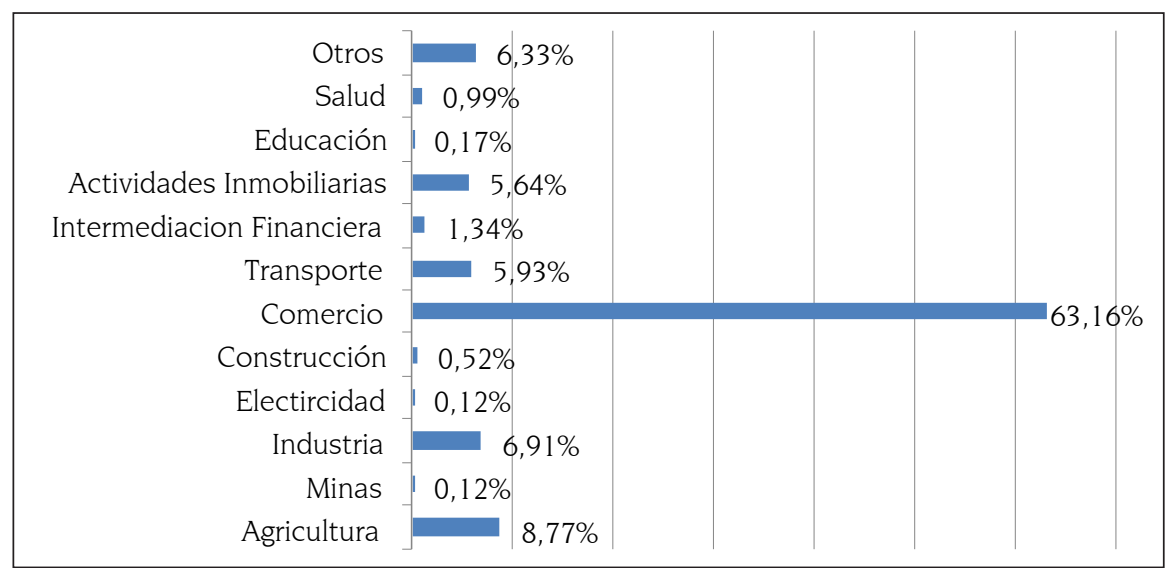

Fuente: elaboración propia. Datos Superintendencia de Sociedades de Colombia y la Cámara de Comercio del Cauca. 
el sector de hoteles, restaurantes, bares y similares, cuyas pérdidas equivalen a \$834.619.753 millones de pesos, todos ellos registrados en Popayán.

La tabla 5 muestra el monto invertido, el monto recibido y el monto perdido por comerciantes en DRFE por sector económico según el código CIIU. De igual forma, se encontró que 2.145 comerciantes registrados en la Cámara de Comercio del Cauca, es decir, el $21.45 \%$ de todos los comerciantes, provenientes de 36 de los 41 municipios del departamento del Cauca, invirtieron en la captadora de dinero DRFE y posteriormente demandaron ante la Superintendencia Financiera por la pérdida de su dinero.

La lista de dichos comerciantes se divide en cuatro partes, las tres primeras corresponde a aquellos cuyas demandas fueron aprobadas por el hecho de haber perdido la totalidad o parte de su inversión, la cuarta parte pertenece a los rechazados, aque- llos que por no haber perdido su inversión les fue rechazada su demanda. Entre los comerciantes cuya demanda fue aprobada se hallaron 943 mujeres y 783 hombres, para un total de 1.723 comerciantes registrados en la Cámara de Comercio que realizaron inversiones en DRFE.

En cuanto a los demandantes que no lograron que su petición fuera aprobada se encontraron 217 mujeres y 202 hombres, para un total de 419 comerciantes inversionistas.

Tabla 5. Comerciantes inversionistas en DRFE. Total municipal y departamental.

$$
t^{*}=\frac{\rho\left(c_{i}, c_{j}\right) \sqrt{T-2}}{\sqrt{1-\left(\rho\left(c_{i}, c_{j}\right)\right)^{2}}} \sim t_{(T-2)}
$$

Fuente: elaboración propia. Datos Superintendencia de Sociedades de Colombia, base de datos comerciantes y CCC.

Tabla 4. Inversiones de comerciantes en DRFE según Código CIIU

\begin{tabular}{|l|rr|rr|rr|}
\hline \multicolumn{1}{|c|}{ Sector } & \multicolumn{2}{c|}{ Monto Invertido } & \multicolumn{2}{c|}{ Monto Recibido } & \multicolumn{1}{c|}{ Monto Perdido } \\
\hline Agricultura, ganadería, caza y pesca & $\$$ & $212.490 .000,00$ & $\$$ & $74.055 .000,00$ & $\$$ & $138.435 .000,00$ \\
\hline Explotación de minas y canteras & $\$$ & $11.000 .000,00$ & & - & $\$$ & $11.000 .000,00$ \\
\hline Industrias manufactureras & $\$$ & $1.274 .153 .402,00$ & $\$$ & $718.465 .700,00$ & $\$$ & $555.687 .702,00$ \\
\hline Suministro de electircidad, gas y agua & $\$$ & $27.855 .000,00$ & $\$$ & $25.330 .000,00$ & $\$$ & $2.525 .000,00$ \\
\hline Construcción & $\$$ & $118.915 .000,00$ & $\$$ & $85.497 .700,00$ & $\$$ & $33.417 .300,00$ \\
\hline Comercio al por mayor y al por menor & $\$$ & $14.338 .500 .621,00$ & $\$$ & $8.161 .086 .044,00$ & $\$$ & $6.177 .414 .577,00$ \\
\hline Hoteles, restaurantes y bares & $\$$ & $1.879 .798 .216,00$ & $\$$ & $1.122 .993 .823,00$ & $\$$ & $756.804 .393,00$ \\
\hline Transporte, Almacenamiento y comunic & $\$$ & $1.25 .555 .689,00$ & $\$$ & $742.494 .365,00$ & $\$$ & $513.061 .324,00$ \\
\hline Intermediación financiera & $\$$ & $345.991 .400,00$ & $\$$ & $217.709 .500,00$ & $\$$ & $128.281 .900,00$ \\
\hline $\begin{array}{l}\text { Actividades inmobiliarias, empresa- } \\
\text { riales }\end{array}$ & $\$$ & $1.351 .082 .516,00$ & $\$$ & $804.794 .950,00$ & $\$$ & $546.287 .566,00$ \\
\hline Educación & $\$$ & $20.070 .000,00$ & $\$$ & $6.800 .000,00$ & $\$$ & $13.270 .000,00$ \\
\hline Servicios sociales y de salud & $\$$ & $321.647 .496,00$ & $\$$ & $222.819 .560,00$ & $\$$ & $98.827 .936,00$ \\
\hline Otras actividades & $\$$ & $1.246 .147 .779,00$ & $\$$ & $701.044 .560,00$ & $\$$ & $545.103 .219,00$ \\
\hline TOTAL & $\$$ & $22.403 .207 .119,0$ & $\$$ & $12.883 .091 .202,00$ & $\$$ & $9.520 .115 .917,00$ \\
\hline
\end{tabular}

Fuente: elaboración propia. Superintendencia de Sociedades de Colombia y Cámara de Comercio del Cauca 
Gráfico 15. Comerciantes inversionistas rechazados en DRFE. Total por género y porcentaje sobre el total.

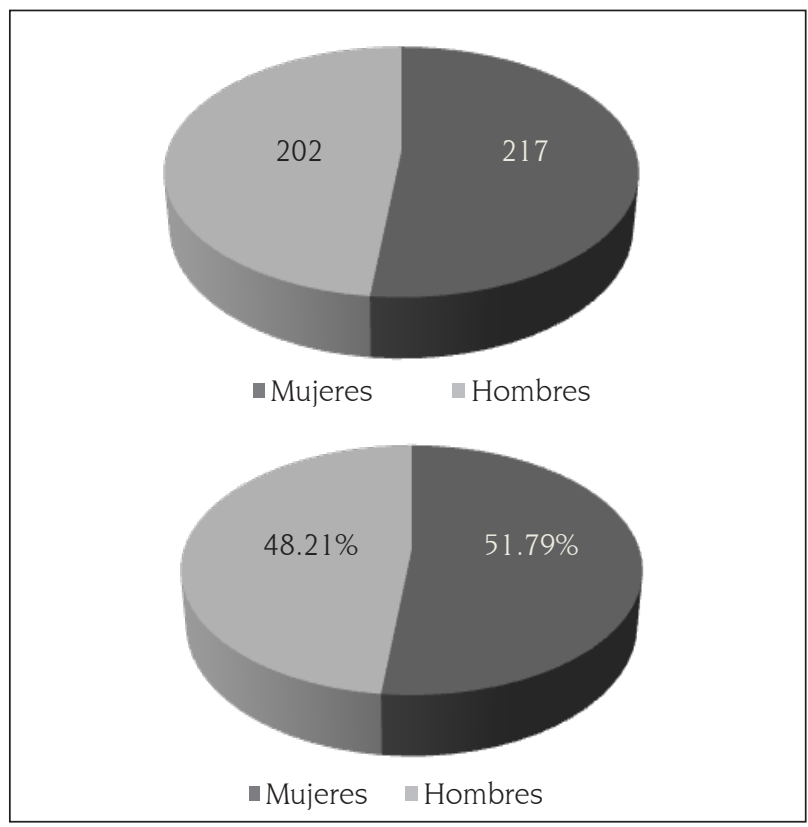

Fuente: elaboración propia. Datos Superintendencia de Sociedades de Colombia, y CCC.

Los comerciantes inversionistas en DRFE pertenecen a 36 municipios del Cauca, pero la gran mayoría están concentrados en la ciudad de Popayán, en donde se alberga el 76,46\% de inversionistas. La tabla 6 indica al municipio donde el comerciante-inversionista tiene su negocio, el número de inversionistas por municipio, y en términos territoriales, el porcentaje de inversionistas dentro del total. El porcentaje de participación de comerciantes adscritos a la Cámara de Comercio del Cauca en los municipios escogidos para el estudio (Patía, Popayán, Santander y Timbío), es el siguiente:

El mayor número de comerciantes- inversionistas en las pirámides se encuentra en Popayán (76.46\%), seguido de Santander de Quilichao (8.78\%), Patía (2.53\%) y Timbío (1.68\%).

\section{CONCLUSIONES}

Se podría decir que a mitad del 2008, mientras el país vivía una situación crítica debido a la desaceleración económica producida por la crisis financiera mundial, la economía de la regional del Cauda tuvo un soporte económico generado por las pirámides que permitió en ese corto período blindar la economía regional del impacto de la crisis financiera. Sin embargo, el desplome de las pirámides generó a final de año un efecto mucho más grave para la economía regional que vio disminuir el PIB a niveles superiores a los nacionales. Debido a la modificación en la estructura productiva del Cauca, que

Gráfico 16. Comerciantes inversionistas según municipios en DRFE. Porcentaje sobre el total departamental.

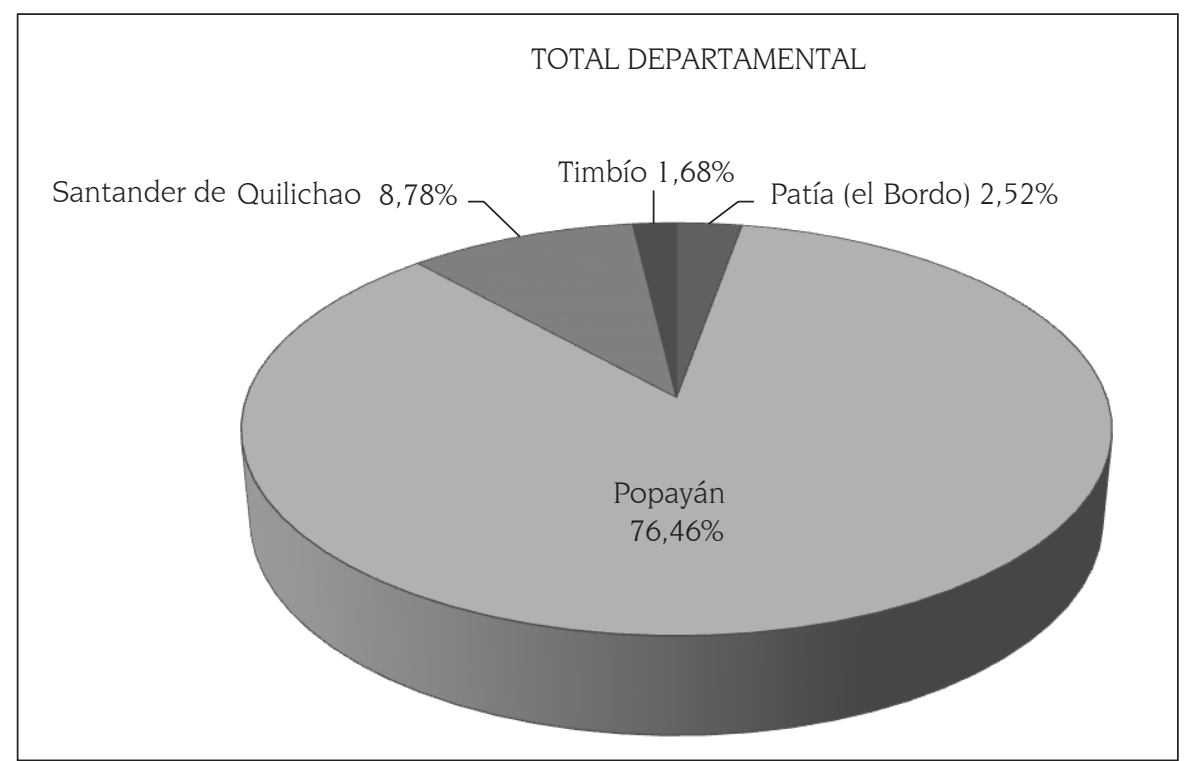

Fuente: elaboración propia: Datos Superintendencia de Sociedades de Colombia, base de datos comerciantes y CCC. 
pasó de ser un departamento netamente agrícola a uno con rasgos fuertes de industrialización (Gómez, 2010, p. 9), la crisis del PIB desde el lado de la demanda agregada no será un fenómeno coyuntural. El PIB caucano, a diferencia del nacional, se caracteriza por tener memoria larga (aproximadamente de 3 períodos), por tanto, se prevé que las lesiones en la estructura productiva se sentirán hasta el 2012.

Las variables económicas regionales que fueron afectadas por la crisis financiera han mostrado en años anteriores que se mueven por encima del PIB regional, a excepción del año 2008. Lo contrario sucede con las variables afectadas por el indicador pirámides, que se comportaron históricamente con un crecimiento por debajo del crecimiento del PIB regional.

En el 2008 el PIB del Cauca muestra un comportamiento de crecimiento positivo y por encima de la crisis financiera, gracias a que el auge de las pirámides amortiguó durante dos trimestres el impacto negativo de la desaceleración generada por la crisis financiera, pero el golpe para la economía regional fue muy fuerte al final del año con el desplome de las pirámides, pues a pesar de que las variables relacionadas con el indicador crisis financiera iniciaban una mejoría, el PIB regional tiene un descenso dramático.

A partir del análisis de la matriz de correlaciones, se evidencia que la crisis financiera no tiene una alta correlación con el PIB del Cauca, a diferencia de la correlación alta y positiva del PIB regional con el indicador pirámides. Es decir, en el comportamiento del PIB regional en el 2008, tuvo mayor incidencia lo sucedido con las pirámides que lo sucedido con la crisis financiera mundial, posiblemente porque esta última afectó regiones del país con grandes participaciones en el mercado de fondos prestables y en el mercado bursátil, a diferencia del Cauca, el cual tiene una participación en estos mercados muy baja, sobre todo en aspectos como la bancarización (Olave, 2010, p. 33).
Los efectos sociales generados por el derrumbe de las pirámides se acentúan en el primer trimestre de 2009. Algunos indicadores sociales relacionados con la salud y la inseguridad muestran un importante deterioro en la región. Las mayores evidencias se encuentran en la enorme disminución de afiliados al régimen contributivo, el incremento de delitos como hurtos y lesiones personales y los testimonios expresados por algunos funcionarios públicos en torno a los impactos en la salud mental de los habitantes de la región. Es muy probable el incremento de la pobreza para el Departamento en el 2009, lo que debe obligar a los Gobiernos local y regional a adoptar medidas económicas y fiscales que minimicen sus efectos en la población más vulnerable, particularmente las mujeres, quienes fueron adicionalmente las mayores afectadas por la caída de las pirámides.

El perfil de los "inversionistas" de las pirámides muestra que las mujeres fueron las más arriesgadas, sin embargo, no fueron las más afectadas. Los comerciantes afiliados a la Cámara de Comercio del Cauca que invirtieron en las pirámides y que perdieron más de 9.5 billones de pesos se ubican fundamentalmente en el sector de comercio al por mayor y al por menor, reparación de vehículos automotores, motocicletas, efectos personales y enseres domésticos y cuyas pérdidas ascendieron a 2.1 billones de pesos. El segundo sector más afectado por la caída de las pirámides es el sector de hoteles, restaurantes, bares y similares, cuyas pérdidas equivalen a 835 millones de pesos, aproximadamente. El 76,46\% de los comerciantes que invirtieron se ubican en Popayán.

Con la construcción de los indicadores se busca rastrear el comportamiento de fenómenos económicos que directamente no son observables, para poderse contrastar con fenómenos que sí lo son, como el ciclo del PIB. La comparación debe ser siempre cualitativa, antes que cuantitativa, pues las unidades de medida son disímiles, y por tanto, deben expresarse de forma normalizada. Este mé- 
todo puede ser replicable en cualquier otra región que carezca de información relevante para analizar un fenómeno.

\section{BIBLIOGRAFÍA}

Asobancaria -Asociación Bancaria- (2008). Informe Financiero Trimestral. Septiembre. Bogotá, 15p.

Banco de la República (2007). Informe de la Junta Directiva al Congreso de La República. Bogotá, marzo, 106p.

Cánovas, Gemma (2008). El oficio de ser mamá. Barcelona: Editorial Paidos, España. 225p.

Caruana, Jaime (2008). Global Financial Stability Report. Financial Stress and Deleveraging: Macro-financial Implications and Policy. Washington D.C., Fondo Monetario Internacional, 180p.

CPC -Consejo Privado de Competitividad- (2009). Informe Nacional de Competitividad 2008-2009. Presidencia de la República de Colombia, Bogotá 319p.

El País (2008). El desplome de las pirámides en Colombia. [En línea] Diario El País, Cali, Colombia, noviembre 16. Disponible en: http://lacomunidad.elpais. com/cosa-publica/2008/11/16/el-desplome-laspiramides-colombia. [Consultado en septiembre de 2009].

El Tiempo (2008). Fiscalía busca dos peces gordos de DMG: Luis Fernando Cediel Rozo y Germán Enrique Serrano Reyes. [En línea] Diario El Tiempo, Bogotá, Colombia, marzo 23. Disponible en: http://www. eltiempo.com/archivo/documento/CMS-4896152. [Consultado en septiembre de 2009].

FENALCO (2009). Bitácora Económica. Presidencia Nacional de Fenalco, marzo, 7p.

Gómez, Mauricio. (2010). Análisis de la productividad multifactorial de la industria manufacturera caucana 1993-2006. Tesis de Maestría en Economía Aplicada, Universidad del Valle, Cali. 52p.

Gómez, Mauricio; Miller, Alejandra; Rivera, Isabel (2006). Análisis y predicción de la economía del Cauca
1960-2005. Proyecto SITEC. Editorial Universidad del Cauca, 160p.

González, Jorge Iván (2008). Desentrañando el misterio de DMG. Razón Pública. [En línea] Bogotá D.C., Razón Pública, Noviembre de 2008. Disponible en: http:// www.razonpublica.com/index.php?option $=\mathrm{com}$ contentEtask=viewEid $=271$ EItemid $=189$. [Consultado noviembre de 2009].

Gujarati, Damodar (2004). Econometría. México: McGraw Hill Cuarta edición, 972p.

Hamilton, J. (1994). Time Series Analysis. Princeton Unievrsity Press. New Jersey: Princeton..

Härdle, W. (1990), Applied Non-parametric Regression. Economertric Theory. Vol 8, No. 3. p. 413-419.

Hodrick, Robert J. y Prescott, Edward C. (1980). Postwar U. S. Business Cycles: An Empirical Investigation. Journal of Money, Credit and Banking, Vol. 29, No. pp. 11-16.

Larrañaga, Mertxe (2009). Mujeres, tiempos, crisis: combinaciones variadas. Revista de Economía Crítica, Segundo Semestre, No. 8, pp. 113-120.

Muñoz A., Jorge Enrique y Perfetti del Corral, Mauricio. (2003) Pronóstico del producto de Caldas con base en la utilización de Indicadores sintéticos incorporados en un Modelo VEC. Manizales: CRECE.

Olave, S. (2010). Influencia de la ley hábeas data en el racionamiento del crédito en el departamento del Cauca: Un modelo de desequilibrio. Tesis. Universidad del Cauca.

Pankratz, Alan (1991), Forecasting with Dynamic Regression Models. New York: John Wiley \& Sons Inc.

Semana (2008). DMG: el imperio contraataca. [En línea] Publicaciones Semana, Semana.com, noviembre 14. Disponible en: http://www.semana.com/economia/dmg-imperio-contraataca/117731-3.aspx. [Consultado en agosto de 2009]. 